\title{
Recent Progress of Nonfullerene Acceptor Molecules for Bulk-Heterojunction Polymer Solar Cells
}

\author{
Qin Xu, ${ }^{a}$ Yan-Mei Tan, ${ }^{a}$ Bao-Yi Ren, ${ }^{*, a, b}$ Meng-Jiao Wang, ${ }^{c}$ Ya-Guang Sun, ${ }^{a}$ and Zhong-Min Su ${ }^{*, b}$ \\ ${ }^{a}$ College of Applied Chemistry, Shenyang University of Chemical Technology, Shenyang, \\ Liaoning 110142, China \\ ${ }^{b}$ School of Chemistry and Environmental Engineering, Changchun University of Science and Technology, \\ Changchun, Jilin 130022, China \\ ${ }^{c}$ Department of Chemistry, Fudan University, Shanghai 200433, China
}

Email: renbaoyi@syuct.edu.cn (B. Y.), zmsu@nenu.edu.cn (Z. S.)

\begin{abstract}
The bulk-heterojunction (BHJ) polymer solar cell (PSC) has been considered as one of the most promising next-generation photovoltaic technologies. Electron-accepting material, which is the key component as important as polymer donor in the BHJ blend of a PSC, can almost only choose from fullerene derivatives before 2015. Currently, nonfullerene acceptor (NFA) materials are attracting drastically increasing interest in organic photovoltaics as alternatives for fullerene derivatives, due to their advantages of structural diversity, low-cost, as well as extraordinary chemical, thermal, and photostability. Benefiting from the facile functionalization of NFA molecules, their photoelectric properties and stacking characteristics can be adequately adjusted to promote photoinduced charge separation and extraction. In recent five years, a lot of NFA molecules have been successfully designed and synthesized, and the power conversion efficiencies of NFA-based PSCs have reached $\sim 13 \%$, revealing the great potential of NFA-material for improving PSCs performances. In this review, the summary and discussion are focused on the molecular architecture and device performance of three types of NFAs materials including PDI-based acceptors, A-D-A and A-ח-D-ח-A conjugated acceptors, aiming to understand the structure-property relationship.
\end{abstract}

Keywords polymer solar cells, bulk-heterojunction, electron acceptor, nonfullerene, structure-property relationship

\section{Introduction}

The researchers' enthusiasm has continued to be motivated by the crisis of energy resource and environmental pollution. To break away from the predicament, photovoltaic technology provides a promising platform. Since 1986, Tang $^{[1]}$ developed a bilayer heterojunction organic solar cell with the power conversion efficiency (PCE) of $\sim 1 \%$, opening up a new way of photoelectric conversion. Subsequently, Heeger and co-workers ${ }^{[2,3]}$ found the photo-induced ultrafast electron transfer from a conducting polymer to fullerene, and then they enhanced the PCE of polymer-fullerene system using a bulk heterojunction $(\mathrm{BHJ})$ structure. The polymer solar cells (PSCs), with the advantages of lightweight, flexibility, large area fabrication and low-cost, represent the future orientation of organic photovoltaics. In the past twenty years, conjugatedpolymer donor materials have been developed abundantly. ${ }^{[4,5]}$ However, the research about acceptor materials has remained relatively stagnant for a considerable long period.

Indeed, fullerene derivatives were the predominant accepter materials for PSCs, until now [6,6]-phenyl- $\mathrm{C}_{61}$-butyric acid methyl ester $\left(\mathrm{PC}_{61} \mathrm{BM}\right.$, Figure 1$)$ is still used as reference substances to illustrate the performance of innovative acceptor materials or as another acceptor component to cover the light absorption of ultraviolet region. This is largely associated with the outstanding feature of fullerene acceptors, such as high electron affinity caused by low-lying LUMO, relatively high electron mobility, and quasi-isotropic charge transport properties owing to a LUMO delocalizing over the whole surface of fullerene. ${ }^{[6]}$ However, the fullerene acceptors possess a series of inherent defects including: (i) weak absorption in the abundant region of the incident solar spectrum, which limits their ability to harvest photocurrent, (ii) limited tunability in terms of spectral absorption, (iii) high synthetic costs, especially for the high performing $\mathrm{C}_{70}$ derivative, and (iv) morphological instability due to fullerene diffusion and aggregation in the thin film over time. ${ }^{[7]}$ Therefore, Indene- $C_{60}$ bisadduct (ICBA), developed by $\mathrm{Li}$ and co-workers, is a rare high-performance fullerene acceptor in comparison with PCBM. $^{[8]}$

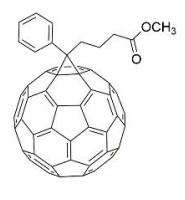

$\mathrm{PC}_{61} \mathrm{BM}$

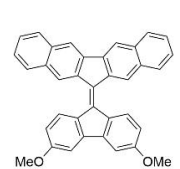

9.9'BF

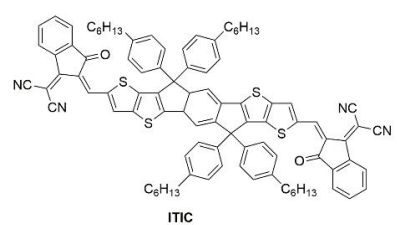

(1)
Figure 1 Examples of acceptor materials for PSCs.

According to recently reports, the PCEs of polymer/ fullerene bulk heterojunctions are $10 \%-12 \%$, closing to the practical maximum efficiency prediction. ${ }^{[9]}$ In order to reach $15 \%-20 \%$ efficiencies for single-junction PSCs, a different approach should be explored.

In fact, using non-fullerene molecules as acceptor materials 
is always keep on researchers' minds. Traditional $n$-type units, such as perylene diimide (PDI), diketopyrrolopyrrole (DPP), and isoindigo (ID), indenofluorene (IF), are tried to introduce into the molecular design of acceptor materials. ${ }^{[10]}$ In addition, extensive efforts have also been paid to develop novel core backbone. ${ }^{[11]}$ For instance, Wudl and co-workers ${ }^{[12]}$ reported a novel 9,9'-bifluorenylidene (9,9'BF, Figure 1) dimers. The novel aromatic backbone can form a 14- 1 -electron fluorenide anion. The corresponding device, using 9,9'BF dimers as acceptor and $\mathrm{P} 3 \mathrm{HT}$ as donor, gave a PCE of $1.7 \%$. Exhilaratingly, an enormous breakthrough for non-fullerene acceptors (NFAs) was reported in 2015. The ITIC molecule (Figure 1) designed and synthesized by Zhan and co-workers showed excellent photovoltaic performance beyond $\mathrm{PC}_{61} \mathrm{BM}$. Both using PTB7-Th as polymer donor, the ITIC-based BHJ device possessed the PCEs as high as $6.80 \%$, which is higher than controlled $\mathrm{PC}_{61} \mathrm{BM}$ device $(6.05 \%) .{ }^{[13]}$ Being inspired by the remarkable work mentioned above, the research about small molecule NFAs have become one of the hottest topics in the field of organic photovoltaics. Precisely because of these progresses, the PCE of single-junction device is more than $14 \%$ now. ${ }^{[14]}$

In recent five years, the NFAs materials have being developed in the field of organic photovoltaics as never before, and the efficiencies of PSCs devices are setting records along with the progress of NFAs, such as its $n$-type units, conjugated backbone, side-chain engineering, etc. In this review, we mainly place our concentration on the rylene contained, A-D-A or A-ח-D-ח-A conjugated NFAs molecules in view of their classical structures, high performance, and distinct structureperformance relationship. Furthermore, the remaining problems and challenges and the key research directions in the near future are also discussed.

\section{The basic electron processes and parameters in PSCs device}

In a typical BHJ device, the active layer possesses bi-continuous interpenetrating network structure to maximize the interface area of donor and acceptor materials, in which three subsequent processes proceeded to generate photocurrent (Figure 2) as follows: (i) The active layer absorbed incident photons to generate electron-hole pairs (excitons) because of the strong Coulomb attraction between the electron and the hole. Then excitons diffuse towards the donor-acceptor interface; (ii) Charge separation, excitons dissociate by forming charge transfer state, in which the charges reside on different molecules. However, they remain bound to each other by Coulombic attraction at D-A interface; (iii) Holes and electrons transport in donor and acceptor phases, and they are collected at the electrodes, respectively.
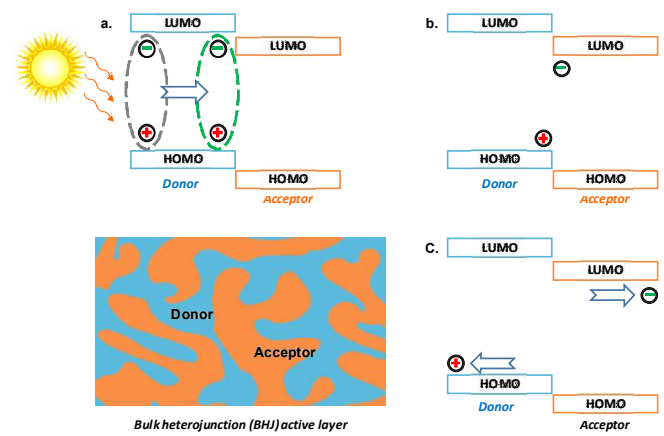

Figure 2 (a) Photon absorption, exciton generation and diffusion, (b) charge separation at D-A interface, and (c) holes and electrons transporting and collection.
The morphology of non-fullerene BHJ layer results from phase separation and crystallization of the blended materials. Electron processes are significantly affected by the nanomorphology. Small domain size provides extensive interface interaction. On the one hand, it caused possibly large charge generation yield; on the other hand, the combination process lead to annihilation of charge carriers. Excessively large domain size with a lowered interface area usually obtains good charge transporting capability, but it lacks of charge generation yield. The ideal domain size is fall in between the two cases above-mentioned, guaranteeing both large charge generation yield and good charge transport.

The performance of organic solar cells can be revealed by their current density $(J)$-voltage $(V)$ characteristics. Figure 3 is a typical $J-V$ curve measured under dark and incident-light illumination conditions. The open-circuit voltage ( $\left.V_{O C}\right)$ represents the maximum voltage when no current flows across the solar cell. The value of $V_{O C}$ depends on the energy gap between the HOMO level of donor molecule and LUMO level of acceptor molecule. $V_{O C}$ is also affected by some factors such as energetic disorder, $\mathrm{CT}$ states, micro-structure of $\mathrm{BHJ}$, and D-A interface area. The recombination process would always lead to the loss of $V_{\mathrm{OC}}{ }^{[15]} \mathrm{J}_{\mathrm{SC}}$ stands for short-circuit current, and it can be related to external quantum efficiency if we integrate the contributions at each different wavelength. $J_{\mathrm{SC}}$ is mainly affected by spectral absorption coverage of donor or acceptor materials. Moreover, the impact of the area of D-A interface in $\mathrm{BHJ}$ device, charge carrier mobility is not negligible. The external quantum efficiency (EQE) can be also called incident photon-to-electron conversion efficiency (IPCE) that is the percentage number of charge carriers collected from the cell per photon shined to the cell. The molar extinction coefficients of the molecular in photoactive layer should be high enough to promote a high EQE. Fill factor $(F F)$ is the ratio that the maximum power delivered by the cell divided by the product of $J_{S C}$ and $V_{\mathrm{OC}}$. The relationship among carrier recombination, relaxation, and transport processes are vital for a high FF. Finally, the PCE $(\eta)$ is defined as showing in Figure 3.

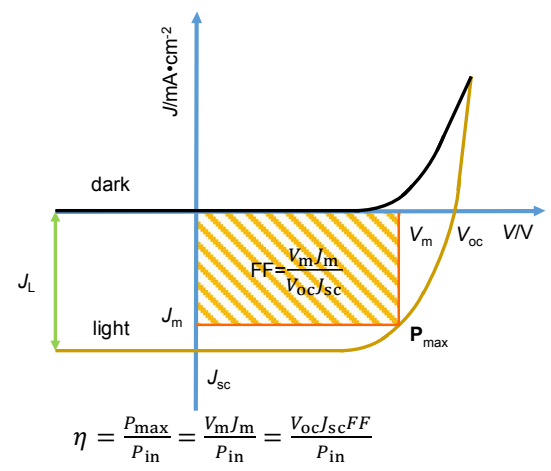

Figure 3 A typical $J-V$ curve measured under dark and incident-light illumination conditions. Essential parameters determining the cell performance are shown: $V_{O C}=$ open-circuit voltage; $J_{\mathrm{SC}}=$ short-circuit current density; $F F=$ fill factor; $V_{\mathrm{m}}$ and $J_{m}$ are voltage and current, respectively, at which the power output of a device reaches its maximum. The power-conversion efficiency $\eta$ is defined as the ratio of maximum power output $\left(P_{\max }\right)$ to power input $\left(P_{\mathrm{in}}\right) . \mathrm{J}_{\mathrm{L}}=$ light-generated current

\section{PDI}

Perylene-3,4,9,10-tetracarboxylic acid diimides (PDI) is a highly planar $n$-type unit with readily available and low cost merits. The $n$-type characteristic of PDI is mainly attributed to its relatively high electron affinity and electron mobility. In addition, 
high extinction coefficient in the visible region and good photochemical stability make PDI attractive candidate for building NFAs molecules. ${ }^{[16,17]}$ As shown in Figure 4, PDI is a planner contain three modification sites: a position $(2,5,8,11$ positions), 'bay' position (1,6,7,12 positions) and imide position. However, the planar aromatic backbone usually arouse strong $\pi-\pi$ stacking, thus PDI and its derivatives can self-assemble into micron sized micro-crystals. ${ }^{[18-20]}$ Hence, much attention has been focused on reducing the excessive agglomeration of PDI-based NFAs through rational molecular design.

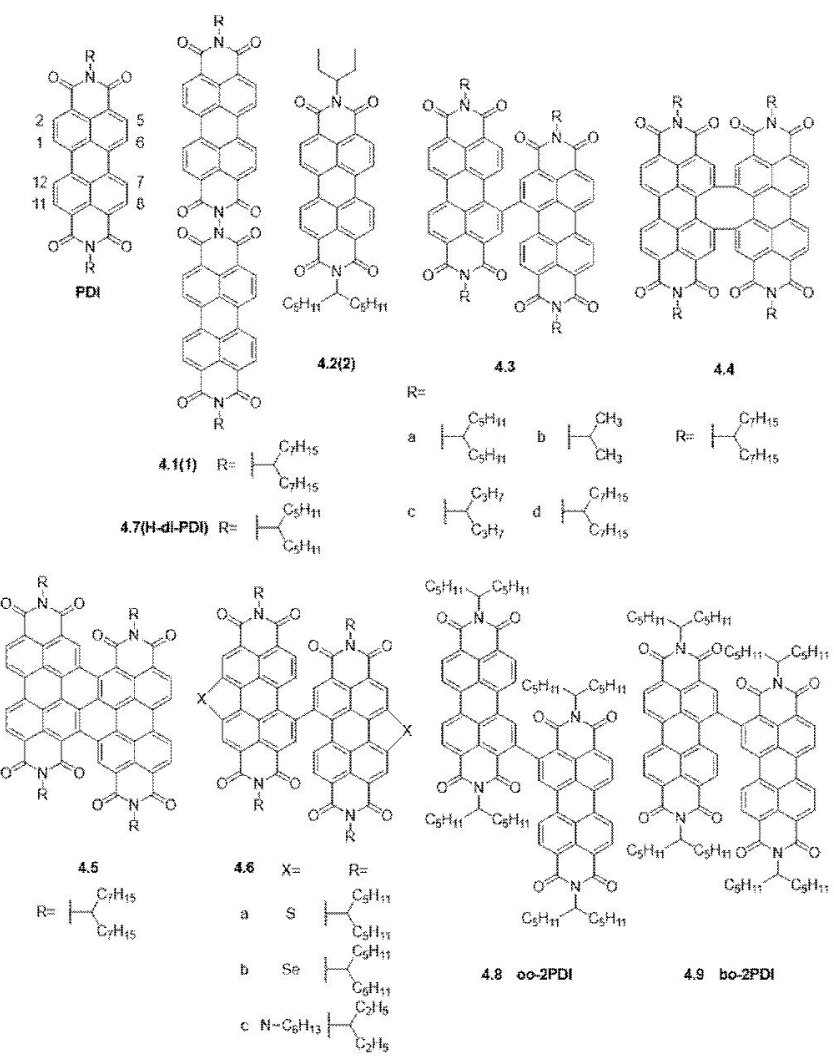

Figure 4 Chemical structures of directly linked PDI dimer.

\section{PDI dimer}

PDI dimers include two typical linking mode: directly connected via $\sigma$-single bond and spacer unit added system. Reducing coplanarity is an effective strategy to avoid the formation of excessively large domains caused by the tendency of over-aggregate. Furthermore, the twist degree between two PDI units can be tuned by the steric effect and threedimensional (3D) structure of added spacers.

\section{Directly linked dimer}

In 2012, Rajaram et al. ${ }^{[21]}$ reported a pioneer work, which introduced a single bond between two PDI units at imide position to obtain an amides dimer. The extraordinary higher $J_{\mathrm{sc}}$ for $\mathbf{4 . 1}$ compared to $\mathbf{4 . 2}$ (Figure 4) was ascribed to the lower planarity of the molecule, in which PDI units are oriented perpendicular to each other. The loss of planarity was posited to lowered phase segregation, expediting charge separation at the D-A interface. The device prepared using PBDTTT-C-T (all of the polymer donors mentioned in this review, see Figure 16) as donor and 4.1 as acceptor showed a PCE of $2.77 \%$.

Jiang et al. ${ }^{[22]}$ prepared a series of dimers with $\mathrm{N}$-alkyl groups. The torsion angle of $s$-diPBI (Figure $4,4.3 \mathrm{c}$ ) is about $70^{\circ}$, which is higher than $d$-diPBI (Figure $4,4.4$ ) and $t$-diPBI (Figure $4,4.5)$. In a $1: 1(w / w)$ blend ratio of PBDTTT-C-T/
s-diPBI based device showed a PCE of $3.63 \%$ with a $V_{O C}$ of $0.73 \mathrm{~V}$, a $J_{\mathrm{SC}}$ of $10.58 \mathrm{~mA} / \mathrm{cm}^{2}$, a $F F$ of $46.80 \%$. The best solvent mixture used for the device fabrication was ternary solvent mixture of DCB (dichlorobenzene) $1.5 \%$ DIO (1,8-diiodooctane)/1.5\% CN (1-chloronaphthalene)

Later, Sun and Meng employed successively heteroatom annulated strategy to synthesize SdiPDI-S andSdiPBI-Se (Figure 4, 4.6a and 4.6b). The dihedral angles of SdiPDI-S and SdiPBI-Se between the PDI planes were determined to be $80^{\circ}$ and $77^{\circ}$, respectively, which are more big than 4.3c (dihedral angle of $\left.67^{\circ}\right){ }^{[23,24]}$ The increase in dihedral angles revealed stronger steric hindrance, stemming from the introduction of the $\mathrm{S}$ and Se bridges. The PSCs with PDBT-T1 as donor achieved a PCE of $7.16 \%$ for SdiPDI-S, and $8.42 \%$ for SdiPBI-Se. $4.6 \mathrm{c}$ can be synthesized via large-scale, column chromatographyfree procedure. Using inverted device configuration (ITO/ZnO/ P3TEA:4.6c/ $/ \mathrm{V}_{2} \mathrm{O}_{5} / \mathrm{Al}$ ), the PSCs gave a remarkable PCE of $7.55 \%$.

In 2015, Wu et al. ${ }^{[25]}$ investigated systematically the influence of molecular geometry of polymer donor for the active-layer morphology of the PSC using B-di-PDI (Figure 4, 4.3c) as acceptor material. 4.3c showed a better miscibility with PTB7 and PTB7-Th. Thus in both case, the devices of B-di-PDI showed high PCEs, $5.00 \%$ for blended with PTB7 and 5.56\% for blended with PTB7-Th. However, the combination of PTB7/H-di-PDI (Figure 4, 4.7) showed a poor PCE of $1.69 \%$. Surprisingly, the blends of PTB7-Th and $\mathbf{4 . 7}$ gave a PCE as high as $6.41 \%$. The result is attributed to the high rigidity and strong $\pi-\pi$ stacking of PTB7-Th relative to 4.7 acceptor.

In 2017, Wang et al. ${ }^{[26]}$ developed three PDI dimers. The dihedral angles between the two PDI monomers are $70^{\circ}, 67^{\circ}$ and $87^{\circ}$ for $b b$-2PDI (Figure 4, 4.3a), oo-2PDI (Figure 4, 4.8) and bo-2PDI (Figure 4, 4.9), respectively. Among them, oo-2PDI was connected directly by the single bond via the $\alpha$-position of the PDI monomers. oo-2PDI exhibited much stronger absorption in the visible region. When blend with PTB7-Th, the formation of favorable morphology had been studied. The PSC device prepared with the blend of PTB7-Th:oo-2PDI (1:1, with $1 \%$ DIO as the additive) displayed an impressed PCE of $8.30 \%$ with a $J_{\text {SC }}$ as high as 18.79 $\mathrm{mA} / \mathrm{cm}^{2}$.

\section{Linker and conformation}

In 2013, Zhang et al. ${ }^{[27]}$ unveiled that thiophene can be introduced as a spacer for linking PDI units at bay region. Calculation result shows the dihedral angles between the PDI-thienyl-PDI planes are $50^{\circ}-65^{\circ}$. By the joint effort of alkoxy group, $\mathrm{N}$-alkyl groups and spacer, the aggregation was significantly reduced compared to monomeric PDI. Thus, bis-PDI-T-EG (Figure 5, 5.1a) yielded smaller phase domains with a size of $12 \mathrm{~nm}$ blended with polymer donor. Accordingly, the PCE of the PBTTT-C-T/5.1a blends was improved to $4.03 \%$. Later, Yan et al. ${ }^{[11]}$ synthesized dozens of bay-linked dimer by employing 6 different arylene spacers, including 5.2-5.6 (Figure 5), to verify the relationship between spacer and phase separation caused by difference of intermolecular aggregation. The result indicated that three-dimensional structures suppress effectively self-aggregation and crystallization of the PDI units. Thanks to the favorable size of domains in blend with $\mathrm{P} 3 \mathrm{HT}$, the PCEs of devices containing spirobifluorene-2,7-diyl and spirobifluorene-2,2'-diyl spacers (Figure $5, \mathbf{5 . 6}$ a and $\mathbf{5 . 6}$ ) reached $2.28 \%$ and $2.35 \%$, respectively.

In 2014, Lin et al. synthesized indaceno[1,2-b:5, 6- $b$ ']dithiophene (IDT) bridge to link two PDI units; that is IDT-2PDI (Figure 5, 5.7). ${ }^{[28]}$ A dihedral angle of $49.6^{\circ}$ between the IDT 

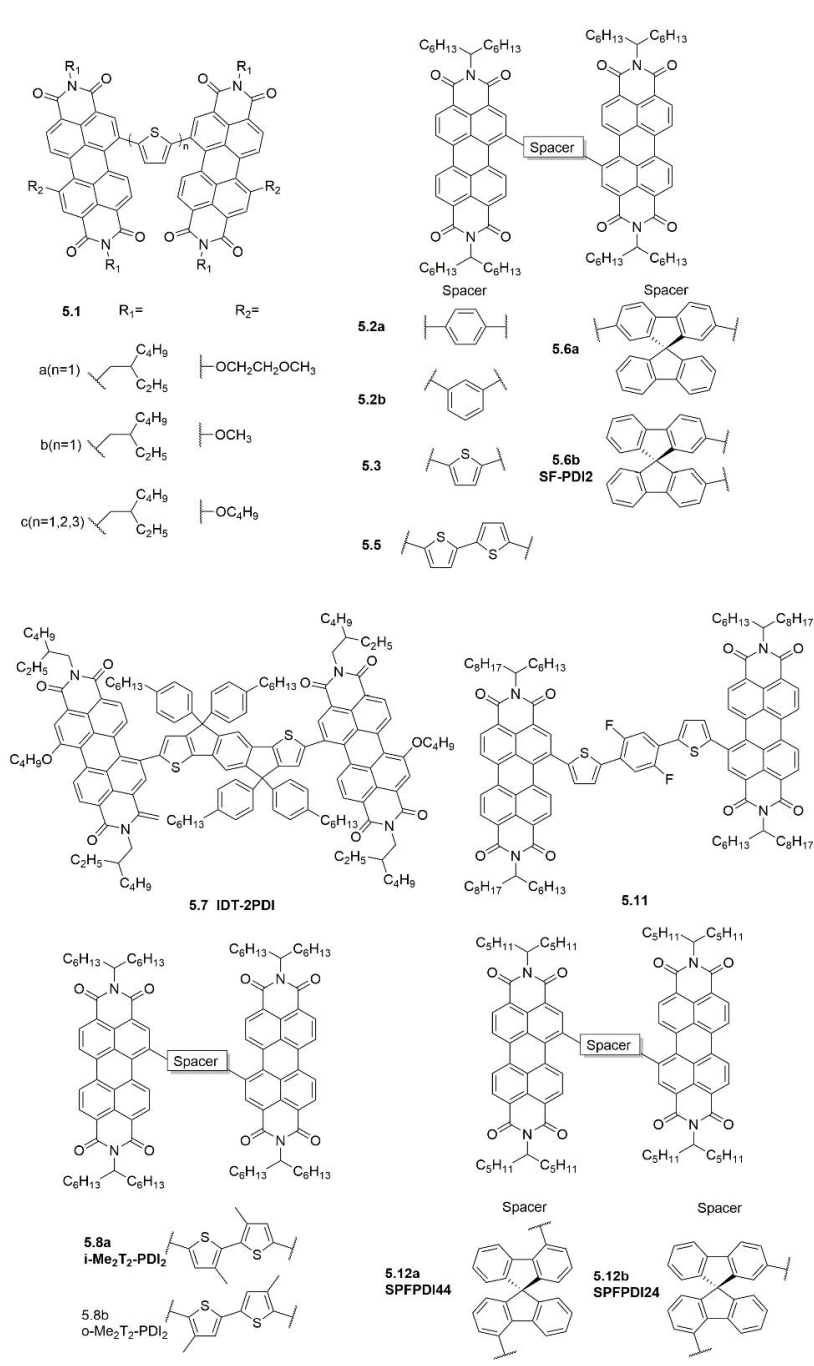

Figure 5 Chemical structures of spacer linked PDI dimer.

and PDI planes had been acquired by the steric hindrance of bulky fused-ring. The blend of P3HT or BDT-2DPP:IDT-2PDI was subsequently fabricated with a structure of ITO/ PEDOT:PSS/BHJ/Ca/Al. The balanced charge transporting ability leaded to a high FF close to 0.67 for the former. Due to complementary absorption and suitable energy levels for the latter, a PCE of $3.12 \%$ with a high $V_{\mathrm{OC}}$ of $0.95 \mathrm{~V}$, a $J_{\mathrm{SC}}$ of 7.75 $\mathrm{mA} / \mathrm{cm}^{2}$, and a $F F$ of 0.42 was achieved.

In 2015, Zhao et al. ${ }^{[2]}$ designed two bridged bithiophenecontaining dimer with methyl at different position. The angles between the two PDI planes are about $60^{\circ}$ for $i-\mathrm{Me}_{2} \mathrm{~T}_{2}-\mathrm{PDI}_{2}$ (Figure 5, 5.8a) and $90^{\circ}$ for $\mathrm{o}-\mathrm{Me}_{2} \mathrm{~T}_{2}-\mathrm{PDI}_{2}$ (Figure 5, 5.8b). Torsion angle of the bithiophene is $54^{\circ}$ and $17^{\circ}$ for $i-\mathrm{Me}_{2} \mathrm{~T}_{2}-\mathrm{PDI}_{2}$ and $\mathrm{O}-\mathrm{Me}_{2} \mathrm{~T}_{2}-\mathrm{PDI}_{2}$, respectively. The domain size of PffBT4T-2DT/o- $-\mathrm{Me}_{2} \mathrm{~T}_{2}-\mathrm{PDI}_{2}$ is clearly larger than that of PffBT4T-2DT/i-Me $\mathrm{M}_{2}-\mathrm{PDI}_{2}$, which is likely due to the different molecular geometries of the two molecules. In addition, the two molecules show different absorption edges. $0-\mathrm{Me}_{2} \mathrm{~T}_{2}-\mathrm{PDI}_{2}$ exhibited a more extended absorption range. Eventually, the spacer in head to head configuration formed smaller domain size and enhanced the aggregation of PffBT4T-2DT in the blend film. Therefore, PSCs based on PffBT4T-2DT/i-Me $\mathrm{T}_{2} \mathrm{~T}_{2}-\mathrm{PDI}_{2}$ exhibited a promising efficiency of $4.1 \%$, while PffBT4T-2DT/ o- $\mathrm{Me}_{2} \mathrm{~T}_{2}-\mathrm{PDI}_{2}$ showed an inferior performance of $3.1 \%$.

In the same year, Wang et al. ${ }^{[30]}$ appended oligothiophene as spacer in different length. $\mathrm{P}(1-3)$ TP (Figure 5, 5.1c) showed smaller dihedral angles of $52.3^{\circ}, 50.7^{\circ}$ and $51.3^{\circ}$, respectively.
Enhanced extinction coefficients, increasing in HOMO level and relatively stable LUMO level contribute to their lower bandgaps. When blend with PBDTTT-C-T, the P(1)TP with moderate planarity produced led to low carrier mobility and PCEs under the same condition. Therefore, a favorable phase separation has been preserved in PBDTTT-C-T/P(1)TP device and the PCE improved to $3.61 \%$.

In 2016, Hadmojo and co-workers ${ }^{[31]}$ designed a 2,5-difluorobenzene inserted dithiophene groups. Interestingly, the $\mathrm{S}-\mathrm{F}$ interactions caused a high planarity in F2B-T2PDI (Figure 5, 5.11). The angle between the two PDI units is almost $90^{\circ}$. The PTB7-Th/F2B-T2PDI based film showed an optimized morphology, which could facilitate electron transport property. Thereby, the devices using F2B-T2PDI as acceptor showed improved PCE over $5 \%$.

Later, Liu et al. ${ }^{[32]}$ gave a detailed investigation of the novel polymer P3TEA combined with non-fullerene acceptor SF-PDI2 (Figure 5, 5.6b). By changing the donor to PffBT4T-2DT, the resulting device displayed a significantly higher driving force, and they also performed exciton dynamics proved the existence of ultrafast charge separation. The raising electroluminescence EQE value of the four blends matched well with the decreasing driving force and revealed a small radiative recombination loss. The solar cell showed the internal quantum efficiency is close to $90 \%$, a satisfactory PCE $9.5 \%$ and a $V_{\text {oc }}$ of $1.11 \mathrm{~V}$ were achieved.

Zhou et al. ${ }^{[14]}$ synthesized two isomeric NFAs based on perylene diimide with 4,4'-/2,4'-substituted spirobifluorene. The steric hindrance is larger when the PDI attached at 4-position compared with the 2-position isomer. The dihedral angles between the two PDI units in SPFPDI44 (Figure 5, 5.12a) and in SPFPDI24 (Figure 5, 5.12b) are $49^{\circ}$ and $60^{\circ}$. The DFT calculation indicated that the 4-position substitution enhanced the twist between the PDI unit and spirobifluorene, while the 2-position substitution extended the conjugation. After the application of $2 \% \mathrm{CN}$ as additive, the devices based on PTB7-Th/SPFPDI44 and PTB7-Th/SPFPDI24 achieved the PCEs of $4.19 \%$ and $4.56 \%$, respectively.

\section{PDI trimer and tetramer}

The structural diversity stems from the connectivity between spacer and PDI. Thus, PDI-derived trimer and tetramer by introducing multiple connector had been done to form a 3D or Quasi-3D conformation. Such a conformation provides a promising approach to suppress aggregation and to produce isotropic. Lin et al. ${ }^{[33]}$ reported a novel star-shaped acceptor using a triphenylamine (TPA) as core. The compound S(TPA-PDI) (Figure 6, 6.1) was synthesized through a Suzuki coupling reaction. The quasi-3D structure can restrain molecular aggregation and facilitate miscibility with donors. While the presence of unbalanced charge carrier mobility and the lack of electron mobility make no contribution to FF. Solution-processed PSCs based on a PBDTTT-C-T/S(TPA-PDI) $(1: 1, w / w)$ blend film with $5 \%$ DIO solvent additive exhibited a PCE of $3.32 \%$.

In 2016, Li et al. ${ }^{[34]}$ added three PDI monomers to the 1,3,5-position of benzene ring. The molecular $\mathrm{B}(\mathrm{PDI})_{3}$ (Figure 6 , 6.2) possess $51.4^{\circ}$ dihedral angle between a PDI moiety and the benzene ring. Hence, the strong crystallization of PDI units were successfully reduced by a congested molecular design, meanwhile, a weak intermolecular interaction maintained among PDI units to form a short-range stacking. The best device based on PTB7-Th/B(PDI) 3 gives a PCE of $5.65 \%$ at a $V_{\mathrm{OC}}$ of $0.83 \mathrm{~V}, \mathrm{a} J_{\mathrm{SC}}$ of $13.12 \mathrm{~mA} / \mathrm{cm}^{2}$, and a $F F$ of 0.52 .

Liu and co-workers ${ }^{[35]}$ reported an acceptor bearing four PDI groups. The highly twisted nonplanar 3D molecular 


\section{General Chemistry}

structure of $\mathrm{TPE}-\mathrm{PDI}_{4}$ (Figure 6, 6.4) mainly results from high steric hindrance among tetraphenylethylene (TPE) core and PDI. They also supported three possible non-equivalent geometrical conformers, which contains "edge-to-face" and "edge-to-face" geometry. In this case, favorable morphology and high electron mobility resulted a high PCE of $5.53 \%$ when the acceptor was blended with PffPT4T-2DT. The authors effort to append four PDI unites onto pyrazine, aiming at lower the stress of twisting. The TPPz-PDI 4 (Figure 6, 6.5) has a $40^{\circ}$ dihedral angle between the pyrazine unit and the phenyl groups, indicating that pyrazine is a weaker twisting spacer than TPE. After the formation of film, the favorable conformation makes the electron mobility of this material comparable to that of fullerene acceptors. Therefore, the Pffbf-T3(1,2)-2/TPPz-PDI based devices exhibit highest $J_{\mathrm{SC}}$ and $F F$, achieving a PCE as high as $7.1 \%$.

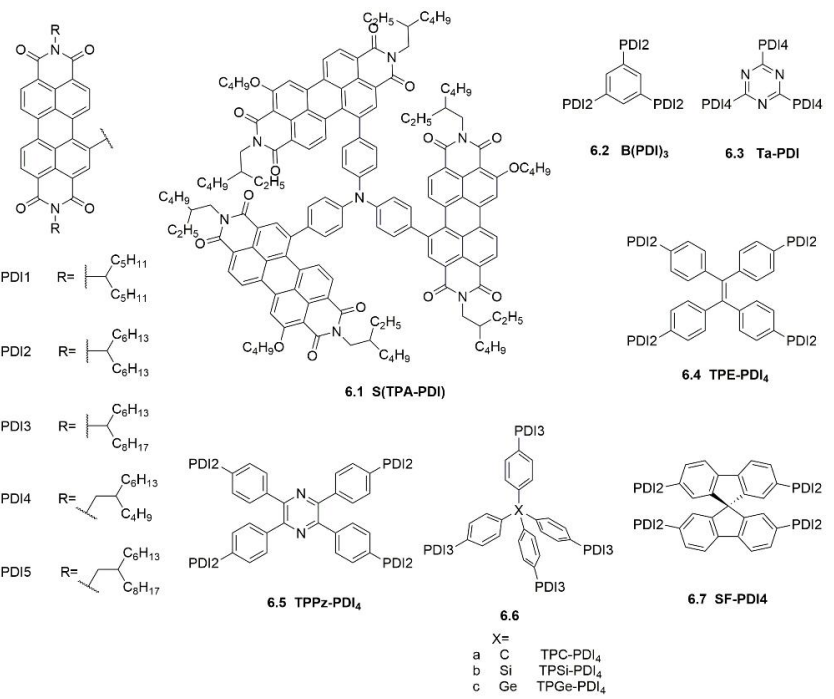

Figure 6 Chemical structures of PDI trimer and tetramer.

By using different central atom of the tetrahedron core, TPC-PDI ${ }_{4}, \mathrm{TPSi}^{-P D I}$, and $\mathrm{TPGe}_{4} \mathrm{PDI}_{4}$ (Figure 6, 6.6a-6.6c) had been designed and synthesized. ${ }^{[36,37]}$ TPSi-PDI $_{4}$ pristine film has one order higher electron mobility than that of mono-PDI pristine film. The germanium analogue TPGe-PDI 4 had also been designed to better understand the size difference of core atom. While TPGe-PDI 4 electron mobility is in the same magnitude order to mono-PDI pristine film. Compared to

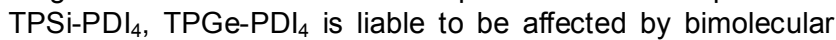
recombination and lower charge dissociation. The PffBT4T-2DT, TPC-PDI ${ }_{4}$ and TPSi-PDI ${ }_{4}$ based PSCs all achieved PCE higher than $4 \%$, while PffBT4T-2DT/TPGe-PDI 4 blends only obtained a poor PCE of $1.6 \%$.

Lee et al. ${ }^{[38]}$ decorated 9,9'-spirobifluorene with PDI in $2,2^{\prime}, 7,7^{\prime}$ positions. The two fluorene units in SF-PDI4 (Figure 6 , 6.7) were perpendicular to each other. The skeleton of the attached PDI show an $18^{\circ}$ twisted angle. The nature of aggregation/crystallization of PDI molecules was significantly alleviated. The interpenetrating $\mathrm{BHJ}$ morphology in the PV4T2FBT/SF-PDI4 device yielded a high $J_{\mathrm{SC}}$ of $12.02 \mathrm{~mA} / \mathrm{cm}^{2}$ with a reasonably high $V_{O C}$ and $F F$, leading to a PCE of $5.98 \%$.

\section{Fused- and spiro-PDI}

In fact, the emphasis has been placed on structures correlating with twisted geometries or extended molecular orbital surfaces. A rich variety of structural possibilities is revealed by fused-ring and embedded spiro-atom to PDI systems.

In 2014, Zhong and co-workers designed and synthesized helical PDI 1 (Figure 7, 7.1) by bay-position fusing. The helical PDI 1 featured a strong absorption from 350 to $550 \mathrm{~nm}$ with a maximum molar extinction coefficient of $1.1 \times 10^{5} \mathrm{M}^{-1} \cdot \mathrm{cm}^{-1}$. Ultrafast electron transfer from PTB7 to helical PDI 1 and hole transfer from helical PDI 1 to PTB7 with a time constant of $\sim 0.2$ ps had been manifested by using transient absorption spectroscopy. ${ }^{[39]}$ After optimization, the PSCs showed the following characteristics: $J_{\mathrm{SC}}=13.3 \mathrm{~mA} / \mathrm{cm}^{2}, V_{\mathrm{oc}}=0.803 \mathrm{~V}, \mathrm{FF}$ $=56.6 \%$, and PCE $=6.05 \%$. Later, they gave the comparison compounds by the fusion of bay linked spacer furan, thiophene and selenophene (Figure 7, 7.2a-7.2c) ${ }^{[40]}$ Among the three NFAs, F-PDI-T (7.2a) has the most ordered packing. The twist angle of F-PDI-T (7.2b) is between those of F-PDI-F and FPDI-Se (7.2c), indicating that the twist angle between the PDI planes plays a critical role in determining crystallinity and molecular packing. The thiophene-containing acceptor F-PDI-T combined with PTB7-Th reached a PCE of $6.72 \%$. Hartnett et al. ${ }^{[11]}$ had prepared thiophene, phenylene and thienothiophene containing PDI analogue. Upon ring fusion, the dimers showed restrained geminate recombination and raised LUMO energy level.

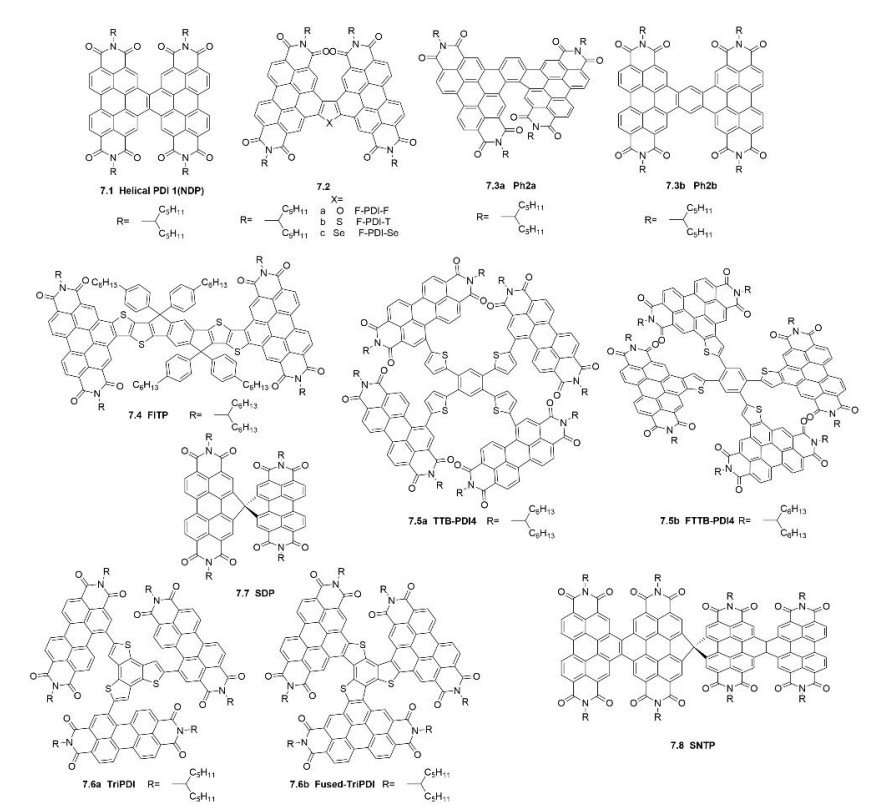

Figure 7 Chemical structures of fused and spiro PDI derivatives.

In 2016, Li et al. ${ }^{[42]}$ developed a fused PDI molecule with indacenodithieno[3,2-b]thiophene (IDTT) with another sidechains in order to suppress the strong intermolecular aggregation. FITP (Figure 7, 7.4) owns a planar molecular backbone, and four hexylphenyl side-chains stretch out titled to the conjugated backbone plane. Compared with common PDI derivatives, the more red-shifted absorption of FITP film lies in the range of $300-700 \mathrm{~nm}$, the strong electron-donating nature of the IDTT result in a higher LUMO of FITP $(-3.75 \mathrm{eV})$ allows a high $V_{o c}$ for PSCs. The addition of $C N$ can't enhance the crystallinity of FITP, but it may influence the orientation of FITP and increase the crystallinity or face-on orientation of PTB7-Th. By blending with PTB7-Th, FITP exhibits good performance with a high efficiency of $7.33 \%$ and a high $V_{\text {oc }}$ of $0.99 \mathrm{~V}$.

In 2017, Zhang et al. ${ }^{[43]}$ reported two novel NFAs, non-fused PDI-tetramer TTB-PDI4 (Figure 7, 7.5a) and fused PDI-tetramer FTTB-PDI4 (Figure 7, 7.5b). The overall geometry of FTTB-PDI4 resembles a "double-decker" structure, which is distinctively different from the unfused analog. The unique geometry of FTTB-PDI4 enhanced both intramolecular and 
intermolecular interactions. Consequently, when employing P3TEA as donor, FTTB-PDI4 exhibited an electron mobility of $(1.1 \pm 0.2) \times 10^{-4} \mathrm{~cm}^{2} \cdot \mathrm{V}^{-1} \cdot \mathrm{s}^{-1}$, which is an order of magnitude higher than TTB-PDI4. X-ray scattering (GIWAXS) profiles of the two blends showed that FTTB-PDI4 exhibits a slightly larger (010) coherence length than TTB-PDI4 (1.5 vs. $1.3 \mathrm{~nm}$ ), which demonstrates the improved molecular packing of FTTB-PDI4 in the blends. The P3TEA/FTTB-PDI4 devices achieved the highest PCE of $10.58 \%$ with a small voltage loss of $0.53 \mathrm{~V}$. In comparison, the highest PCE of the P3TEA/TTB-PDI4 devices was only $7.11 \%$. Combining the extended benzotrithiophene (BTT) core with three PDI moieties, Wang et al. ${ }^{[44]}$ synthesized two acceptors: TriPDI (Figure 7, 7.6a) and fused-triPDI (Figure $7,7.5 b)$. TriPDI displays a highly twisted structure with a large dihedral angle over $50^{\circ}$, while the dihedral angle between PDI moiety and BTT is less than $6^{\circ}$ after ring fusing. The greatly increased LUMO level $(-3.73 \mathrm{eV})$ and slightly increased HOMO made the optical band gap of fused-TriPDI $(2.20 \mathrm{eV})$ larger than that of TriPDI $(1.99 \mathrm{eV})$. The electron mobility of fused-TriPDI device is $1.26 \times 10^{-3} \mathrm{~cm}^{2} \cdot \mathrm{V}^{-1} \cdot \mathrm{s}^{-1}$, which is nearly one order of magnitude higher than that of the unfused structure. Both of the blend films show photoresponse in the range of 330 to $580 \mathrm{~nm}$. The PSCs with the PTB7-Th/Fused-TriPDI blend showed a PCE of $6.19 \%$, almost three times higher than that with PTB7-Th/TriPDI one.

Gao et al. ${ }^{[45]}$ prepared a series of $\pi$-fused PDI oligomers spirodiperylenetetraimide (SDP, Figure 7, 7.7), naphthodiperylenetetraimide (NDP) and PDI tetramer (SNTP, Figure 7, 7.8). The optical energy gaps are 2.17 eV for SDP, $2.21 \mathrm{eV}$ for NDP, and $2.182 .18 \mathrm{eV}$ for SNTP. The LUMO energy levels were estimated to be $-3.91,-3.90$ and $-3.95 \mathrm{eV}$ for SDP, NDP and SNTP. Their electron affinities are comparable to classical fullerene electron accepting materials. Atomic force microscopy (AFM, Figure 8) was employed in order to gain insight into the morphology of the BHJ films. The PTB7-Th/PDI blend films showed large domain size and no continuous interpenetrating network, which are unfavorable for the charge generation. However, the other three blends had fiber features and continuous interpenetrating network. Especially, the PTB7-Th: SNTP active layer possessed more uniformed, flat surfaces with the RMS of $1.02 \mathrm{~nm}$ and fiber features, which was beneficial for the charge separation and transporting. Hence, SNTP based PSCs showed a PCE of $7.17 \%$ with a $J_{S C}$ of 15.21 $\mathrm{mA} / \mathrm{cm}^{2}$ and a $F F$ of 0.61 .

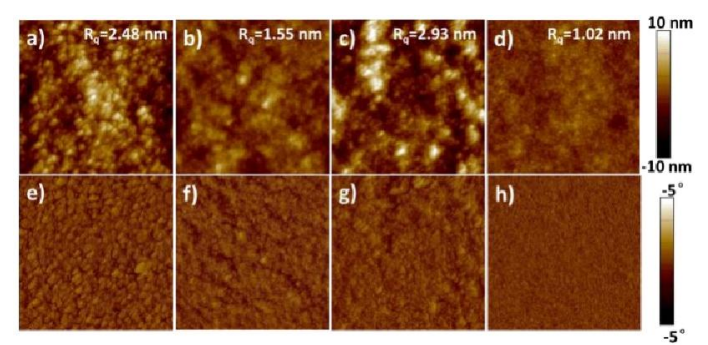

Figure 8 (a) AFM height image $(2 \mu \mathrm{m} \times 2 \mu \mathrm{m})$ of optimized PTB7-Th:PDI, (b) PTB7-Th:SDP, (c) PTB7-Th:NDP and (d) PTB7-Th:SNTP blend films; (e), (f), (g), (h) The corresponding phase images. ${ }^{[45]}$

\section{A-D-A type NFA}

A-D-A systems generally formed using two electrondeficient units (A) end-capped on an electron-rich units (D). The 'push-pull' electron structure allowed to modify the energy levels of frontier molecular orbital and to promote intramolecular charge transfer (ICT). Adjusting the conjugation length of A-D-A structures provide a tunable platform for broadening and enhancing absorption for solar spectra. Besides, the solubility and miscibility can be usually modified by attaching side chains on donor core.

\section{Donor core}

Generally, polycyclic fused-ring arenes and heteroarenes are served as donor unit. Their design and development mainly focused on the chemical modification of fluorene, benzodithiophene (BDT), dithienosilole (DTS), indacenodithiophene (IDT), and dithienopyrrole (DTP). Herein, we discuss and analyze the structure-performance relationship according to the fused-ring number of donor units.

\section{Fused-ring core based on pentacyclic arene}

In 2016, Lin et al. ${ }^{[46]}$ designed a non-fullerene electron acceptor based on indacenodithiophene (IDT), named ICC6IDT-IC (Figure 9, 9.1). The cell using 1:1 weight ratio of PDBT-T1 and IC-C6IDT-IC generated a PCE of $8.71 \%$ without any further treatment, benefiting from their well-matched energy level and complementary absorption. A further discussion relevant to appropriate phase separation, efficient exciton dissociation and negligible bimolecular recombination process in blends also support for the improving of PCE value.

In 2017, Xiao et al. ${ }^{[47]}$ developed a new kind of carbon-oxygen-bridged ladder-type donor core,5,11-dihydrothieno[2',3':5,6]pyrano[3,4-g]thieno-[3,2-c]isochro-mene (CO5). Compared with those of carbon-bridged analogues, the electron-donating capability of $\mathrm{CO} 5$ was improved because of the insertion of electron-rich oxygen atom. At the same time, the increase of atomic numbers in the ring expanded the planarity of backbone, which facilitates carrier transporting. The LUMO level of CO5IC is $-4.13 \mathrm{~V}$, and the optical bandgap of CO5IC is $1.62 \mathrm{eV}$. The PBDB-T/CO5IC devices showed a PCE of $7.49 \%$.

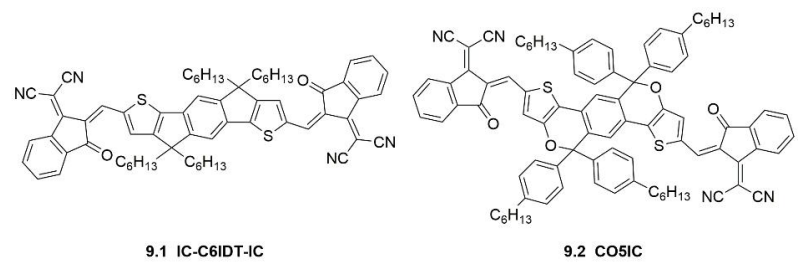

Figure 9 Chemical structures of pentacyclic arene derivatives.

\section{Hexacyclic and heptcyclic arenes as fused-ring core}

In 2017, Ma and coworkers ${ }^{[48]}$ presented two acceptors bearing a fused heterocyclic ring dithienonaphthalene (DTN), DTNIC6 and DTNIC8 (Figure 10, 10.1a and 10.1b). Due to the similarity of their structures, subtle difference in optical bandgap and energy levels had been proven. The length of alkyl chain can adjust the intermolecular interaction. Short alkyl chain promotes intermolecular $\pi-\pi$ interaction, and long alkyl chain increases the solubility meanwhile reducing the $\pi-\pi$ interaction. The device based on PBDB-T/DTNIC6 blend showed a PCE of $3.39 \%$. In contrast, PBDB-T/DTNIC8 one achieved a much higher PCE of $9.03 \%$ with a $J_{S C}$ of $12.92 \mathrm{~mA} / \mathrm{cm}^{2}$ and a $F F$ of $72.84 \%$.

In the same year, Wang et al. ${ }^{[49]}$ designed and synthesized IHIC (Figure 10, 10.2) using the dithienocyclopentathieno[3,2-b]thiophene core and flanked with 1,1-dicyano methylene3 -indanone end-capped units. IHIC has a strong absorption for near-infrared light with an extinction coefficients on the order of $10^{5}$. Space charge-limited current characteristics of the IHIC 


\section{General Chemistry}

film confirmed its electron mobility as high as $2.4 \times 10^{-3}$ $\mathrm{cm}^{2} \cdot \mathrm{V}^{-1} \cdot \mathrm{s}^{-1}$. The HOMO and LUMO energy levels of IHIC were estimated to be -5.45 and $-3.93 \mathrm{eV}$, respectively. The CN-treated PSCs based on the narrow-bandgap polymer PTB7-Th gave a PCE of $9.77 \%$.

Changing fused-position form 1,2,5,6- to 2,3,6,7-pattern of the naphthalene, Zhang et al. ${ }^{[50]}$ developed acceptor IHIC-N (Figure 10, 10.3). IHIC-N exhibits higher ionization energy than IHIC, and the optical bandgap is determined to be $1.66 \mathrm{eV}$. After the addition of $5 \%$ DIO, the PSCs afforded a PCE of $6.91 \%$ with PTB7-Th as donor materials.

Recently, Li et al. ${ }^{[51]}$ developed a novel asymmetric laddertype thiophene-phenylene-thieno[3,2-b]thiophene-fused building block TPTT. The corresponding acceptor TPTT-IC (Figure $10,10.4$ ) can get a PCE of $10.5 \%$ in the PSCs using PBT1-C as the donor. The high $V_{O C}$ value was attributed to the large energy offset between the HOMO of PBT1-C (-5.34 eV) and the LUMO of TPTT-IC $(-3.95 \mathrm{eV})$.

Since 2015, Zhan and co-workers ${ }^{[13]}$ synthesized the acceptor of ITIC (Figure 10, 10.5) using seven member fused-ring indacenodithienodithiophene (IDTT) as core backbone. ITIC showed a strong and broad absorption from visible to near infrared region. The ITIC-based PSCs displayed a $V_{\mathrm{OC}}$ of $0.81 \mathrm{~V}$, a $J_{\mathrm{SC}}$ of $14.21 \mathrm{~mA} \cdot \mathrm{cm}^{-2}$ and a PCE of $6.8 \%$, which is comparable to $\mathrm{PC}_{61} \mathrm{BM}$-based device. When employing PBDB-T as donor, a remarkable PCE of $11.21 \%$ was achieved. ${ }^{[52]}$ Kan et al. ${ }^{[53]}$ developed an isomer of ITIC based on benzo[1,2-b:4,5-b']-dithiophene (BDT) core. The corresponding acceptor NFBDT (Figure 10, 10.6) possesses an optical energy gap of $1.56 \mathrm{eV}$ and FMOs similar with ITIC. Benefiting from highly efficient photo-electron conversion process and suitable phase separation, the PCE of the optimized PBDB-T/NFBDT device reached $10.42 \%$ with a remarkable $J_{S C}$ of $17.85 \mathrm{~mA} / \mathrm{cm}^{2}$.

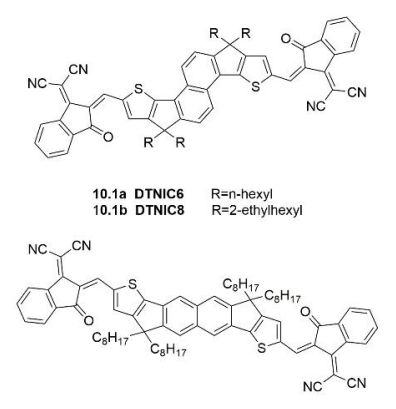

10.3 IHIC-N
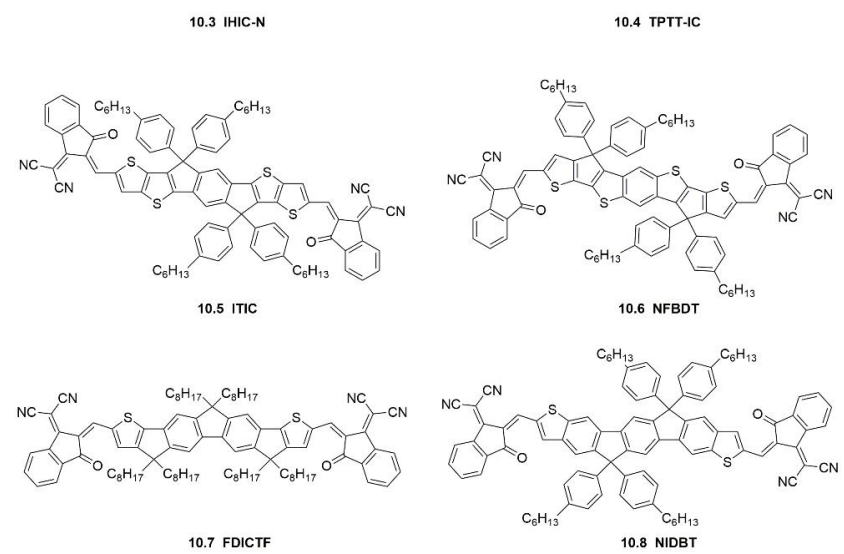

Figure 10 Chemical structures of hexacyclic and heptcyclic arene derivatives.

Fused fluorene with adjacent-thiophene units DICTF, a novel ladder type molecule FDICTF (Figure 10, 10.7) was obtained by Qiu et al. ${ }^{[54]}$ FDICTF possesses a lower bandgap of $1.63 \mathrm{eV}$; the charge carrier mobility were improved and balanced in PBDB-T/FDICTF blend film. The out-of-plane side-chains inhibited effectively over-aggregation so that no clear phase-separation was observed in active-layer. The PBDB-T/FDICTF blended PSCs afforded a PCE of $10.06 \%$.

Selectively replacing two of the thiophenes with benzene rings in IDTT core, a novel donor unit indacenodibenzothiophene (IDBT) was reported by Wang et al. ${ }^{[55]}$ The corresponding acceptor NIDBT (Figure 10, 10.8) shows a wide optical bandgap of $1.84 \mathrm{eV}$. The results of theoretical calculations confirmed the HOMO energy level of IDBT is significantly deeper than that of IDTT. In photo-active layer, the electron and hole mobility was significantly improved. The high electron mobility as well as more balanced charge transporting enhanced $J_{S C}$ and FF of the PSCs device. An optimal PTB7-Th/NIDBT blended film produced a PCE of $4.45 \%$ with a $V_{\mathrm{OC}}$ of $0.831 \mathrm{~V}$, a $J_{\mathrm{SC}}$ of $11.45 \mathrm{~mA} / \mathrm{cm}^{2}$ and a $F F$ of $46.73 \%$.

\section{Octacyclic arene and nonacyclic arene}

In 2017, Zhu et al. ${ }^{[56]}$ synthesized a novel octacyclic nonfullerene acceptor IOIC2 (Figure 11, 11.1). Ascribed to the enlarged NTT core, the electron mobility of IOIC2 is $1.0 \times 10^{-3}$ $\mathrm{cm}^{2} \cdot \mathrm{V}^{-1} \cdot \mathrm{s}^{-1}$, and the HOMO and LUMO levels of IOIC2 are estimated to be -5.41 and $-3.78 \mathrm{eV}$, respectively. After the addition of DIO, the formation of longer $\pi-\pi$ stacking coherence length is benefited to the intermolecular charge transport. As-cast PSCs device based on FTAZ/IOIC2 yielded a PCE of $11.2 \%$, while the device exhibits a PCE of $12.3 \%$ with $0.2 \%$ DIO additive.
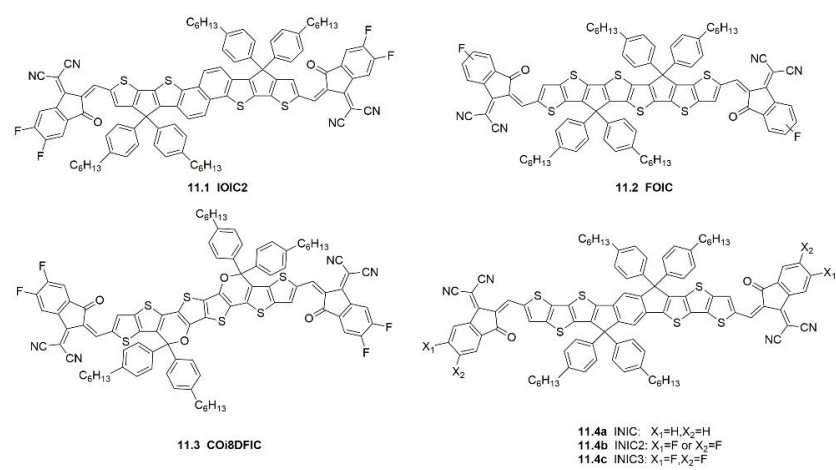

Figure 11 Chemical structures of octacyclic and nonacyclic arene derivatives.

Li et al. ${ }^{[57]}$ designed and synthesized a fused tris(thienothiophene) building block (3TT), in which three thieno[3,2-b]thiophene units were condensed with two cyclopentadienyl rings. FOIC (Figure 11, 11.2) has significantly upshifted HOMO level with slightly downshifted LUMO level, therefore, FOIC exhibits strong NIR absorption from 600 to $950 \mathrm{~nm}$ with extinction coefficient of up to $2 \times 10^{5} \mathrm{M}^{-1} \cdot \mathrm{cm}^{-1}$. The as-cast PSCs based on blends of PTB7-Th/FOIC gave a PCEs as high as $12.0 \%$.

Xiao et al. ${ }^{[58]}$ developed another non-fullerene acceptor COi8DFIC (Figure 11, 11.3) based on carbon-oxygen-bridged ladder-type unit COi8, whose optical band gap is only $1.26 \mathrm{eV}$. The blended film of PTB7-Th/ COi8DFIC has strong absorption in the range of $600-1000 \mathrm{~nm}$. Prepared PSCs of PTB7-Th/ COi8DFIC with $1 \%$ 1,8-diiodooctane (DIO) obtained a PCE of $12.16 \%$ with extremely high $J_{\mathrm{SC}}$ of $26.12 \mathrm{~mA} \cdot \mathrm{cm}^{-2}$.

In 2017, Dai et al. ${ }^{[59]}$ reported several acceptors based on a fused-nonacyclic IBDT core end-capped with non-fluorinated or fluorinated IC. The INIC1-3 acceptors (Figure 11, 11.4) have 
strong absorption in the range of $500-850 \mathrm{~nm}$ with high extinction coefficients about $(2.1-2.5) \times 10^{5} \mathrm{M}^{-1} \cdot \mathrm{cm}^{-1}$. Due to electron-withdrawing property of fluorine, the absorption of the three analogues produce a red shift, while their HOMO/LUMO levels are reduced. Noticeably, FTAZ/INIC3 blend films not only exhibit highest crystallinity compared with other three counterparts, but also maintain both FTAZ and INIC3 semi-crystalline packings. The PSCs based on FTAZ/INIC series exhibit small energy loss of $0.61-0.63 \mathrm{eV}$ and PCEs of $7.7 \%-11.5 \%$, and the best PCE of $11.5 \%$ has been achieved using INIC3 and FTAZ blends.

\section{Polycyclic arene}

Li et al. ${ }^{[60]}$ developed 10-heterocyclic indacenodithiophenoindacenodithiophene. The large-conjugate plane of IDTIDT-IC (Figure 12, 12.1) allows for low reorganization energy and narrow band gap of $1.53 \mathrm{eV}$. Interestingly, PTB7-Th/IDTIDT-IC maintains a EQE of $63 \%$ with small energy loss of $0.59 \mathrm{eV}$. A PCE of $6.5 \%$ was achieved with a high $V_{O C}$ of $0.94 \mathrm{~V}$ based on PTB7-Th/IDTIDT-IC device. They also developed a selenophen analogue IDTIDSe-IC (Figure 12, 12.2) ${ }^{[61]}$ IDTIDSe-IC exhibits a lower band gap of $1.52 \mathrm{eV}$ and strong absorption in the range of $600-850 \mathrm{~nm}$. Combining with a wide band gap polymer J51 as donor, broad absorption coverage from 300 to $800 \mathrm{~nm}$ is obtained, and the corresponding device showed a high PCE of $8.02 \%$ with a $V_{\mathrm{OC}}$ of $0.91 \mathrm{~V}$, a $J_{\mathrm{SC}}$ of $15.16 \mathrm{~mA} / \mathrm{cm}^{2}$ and a $F F$ of $58.0 \%$. Moreover, the EQE of $50 \%-65 \%$ is achieved in the absorption range of IDTIDSe-IC.

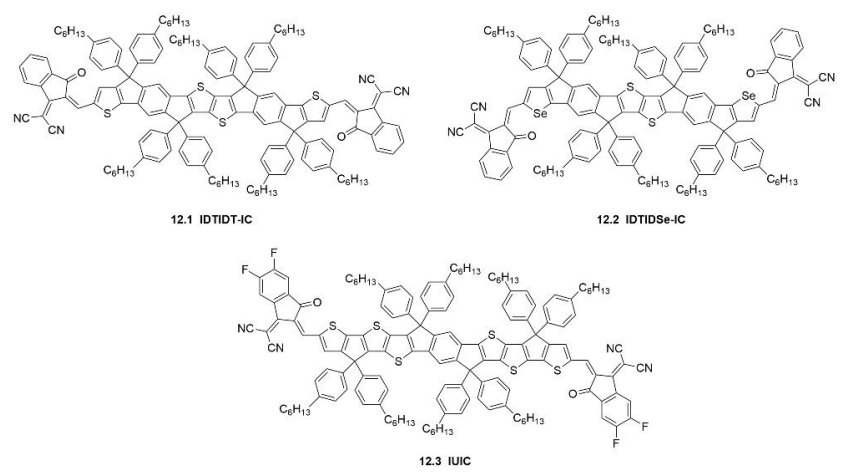

Figure 12 Chemical structures of polycyclic arene derivatives.

Jia et al. ${ }^{[62]}$ designed and synthesized a new fused undecacyclic electron acceptor, IUIC (Figure 12, 12.3), based on a fused-11-ring core IU. IU possesses highly planarity, large $\pi$-conjugation and strong electron-donating ability, which is benefited to photo-harvest and electron mobility. Noncovalent $\mathrm{F}-\mathrm{S}$ and $\mathrm{F}-\mathrm{H}$ bonds are also favorable for enhancing electron mobility. Hence, IUIC shows an optical bandgap of $1.41 \mathrm{eV}$ and red-shifted absorption maximum at $772 \mathrm{~nm}$ with a high extinction coefficient of $3.2 \times 10^{5} \mathrm{M}^{-1} \cdot \mathrm{cm}^{-1}$. The PSCs based on PTB7-Th/IUIC exhibit a PCE of $10.2 \%$. The as-cast one affords a PCEs up to $11.2 \%$.

\section{Side-chain engineering}

Manipulating the side chains on ladder-type core is of critical importance for enhancing the photovoltaic performance, because solubility, intermolecular interaction, chargetransporting property as well as thin-film morphology are significantly influenced by the type, position, and length of side chains.

For instance, Lin et al. ${ }^{[63]}$ replaced the 4-(alkylthio)phenyl chains of ITIC with alkylthienyl, considering that the sulfur atoms are more easily polarized than carbon atoms. Therefore, thienyl side-chains are expected to increase intermolecular interactions, and then facilitate $\pi$-stacking and charge transporting. SCLC results confirmed that ITIC-Th (Figure 13, 13.1) has an electron mobility twice of ITIC. The PTB7-Th/ ITIC-Th and PDBT-T1/ITIC-Th blends show balanced charge transporting and predominant intermolecular $\pi-\pi$ interactions. As a result, the PTB7-Th/ITIC-Th and PDBT-T1/ITIC-Th-based PSCs exhibited PCEs up to $8.7 \%$ and $9.6 \%$, respectively.

Through side chain isomerization, Yang et al. ${ }^{[64]}$ synthesized $m$-ITIC (Figure 13, 13.2). Determined by the SCLC method, the electron mobility of $m$-ITIC $\left(2.45 \times 10^{-4} \mathrm{~cm}^{2} \cdot \mathrm{v}^{-1} \cdot \mathrm{s}^{-1}\right)$ is approximately one and half times of that of ITIC $\left(1.60 \times 10^{-4}\right.$ $\left.\mathrm{cm}^{2} \cdot \mathrm{v}^{-1} \cdot \mathrm{s}^{-1}\right)$. A higher intermolecular self-assembly enhanced the crystalline behavior of the blend film, which are desirable for higher charge carrier mobility thereby higher photovoltaic efficiencies. The PSCs based on $\mathrm{J} 61 / \mathrm{m}$-ITIC show a $V_{\mathrm{OC}}$ of $0.912 \mathrm{~V}$, a $J_{\mathrm{SC}}$ of $18.31 \mathrm{~mA} / \mathrm{cm}^{2}$, a $F F$ of $70.55 \%$ and a high PCE of $11.77 \%$, which is superior to the corresponding PSCs with ITIC as acceptor.

Zhang et al. ${ }^{[65]}$ reported a series of new non-fullerene acceptors including ITIC-SC6, ITIC-SC8and ITICSC2C6 (Figure 13, 13.3a-13.3c). The acceptor with branched side chains has a slightly shallower LUMO level and a wider bandgap than those with linear ones. The charge carrier mobility was gradually increased in the order of ITIC-SC6, ITIC-SC8 and ITIC-SC2C6. In contrast to ITIC-SC6, some fibrillar structures with nanoscale phase separation were formed in the PBDB-ST/ITIC-SC8 blend film, while more significant nanofibers could be clearly observed in the PBDB-ST/ITIC-SC2C6 blend film, suggesting that the side chains of non-fullerene acceptors exert a remarkable influence on the nanoscale morphology of active layers.

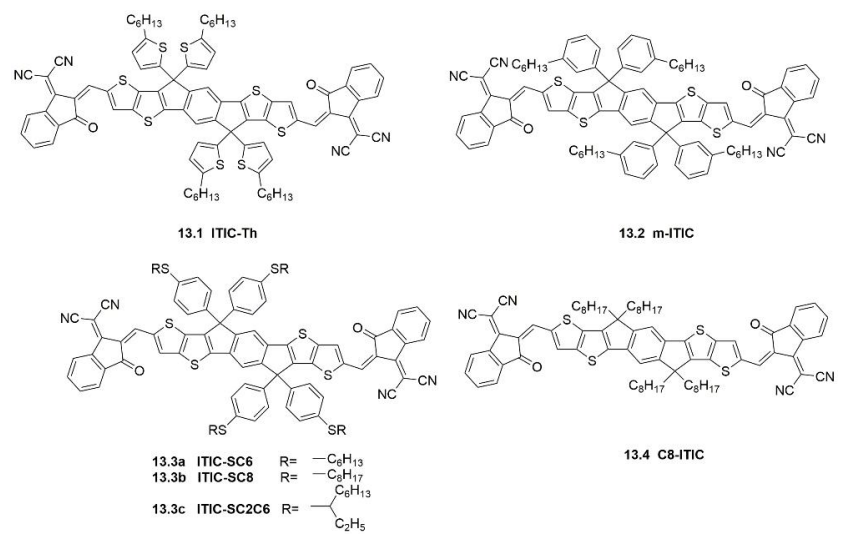

Figure 13 Chemical structures of side chain modified NFAs.

Fei et al. ${ }^{[6]}$ report an alkylated IDTT-based acceptor C8-ITIC (Figure 13, 13.4). The as-cast PFBDB-T/C8-ITIC blend-based PSCs exhibited the best performance with a $J_{S C}$ of $19.6 \mathrm{~mA} / \mathrm{cm}^{2}$, a $V_{O C}$ of $0.94 \mathrm{~V}$, a $F F$ of 0.72 , and an upper PCE of $13.2 \%$. The GIWAXS pattern of C8-ITIC suggested the presence of multiple polymorphs. GISAXS measurement was also used to reveal that the crystallization of both ITIC and C8-ITIC is suppressed by the donor polymers in the blends.

\section{End-capped acceptor units}

The end-capping unit is essential for the overall electron affinity and bandgaps. Reasonable selection and modification of the acceptor unit will directly affect bandgap to maximize $J_{\mathrm{sc}}$ and to adjust the position of LUMO level. The most versatile acceptor unit at the moment is the $1 H$-indene-1,3(2H)-dione (IN). The researchers mainly modify the IN unit through the following three ways: (i) appending weak electron-donating 


\section{General Chemistry}

group (EDG) like methyl ring or appending electron-withdrawing group (EWG) like fluorine on benzene ring, (ii) further fusing the aromatic ring on the benzene ring or replace benzene ring by other aromatic ring, and (iii) one of the original carbonyl oxygen atom in IN unit is completely replaced by dicyanomethylene like 2-(3-oxo-2,3-dihydroinden-1-ylidene)-malononitrile (INCN).

Methyl, a weak and very small electron-donating substituent, could be an ideal candidate to lift the energy levels up slightly, without causing too much steric hindrance for intermolecular packing. Li et al. ${ }^{[67]}$ introduced one and two methyls to acquire acceptors IT-M and IT-DM (Figure 14, 14.1a, 14.1b), respectively. Compared with ITIC, the HOMO and LUMO levels of IT-M and IT-DM are slightly increased, meanwhile, they also exhibit slightly larger bandgaps of 1.60 and $1.63 \mathrm{eV}$. The best device performance was achieved using PBDB-T/IT-M blends, which showed a PCE of $12.05 \%$ with a $V_{O C}$ of $0.94 \mathrm{~V}$, a $J_{S C}$ of $17.40 \mathrm{~mA} / \mathrm{cm}^{2}$, and a $F F$ of $73.5 \%$.
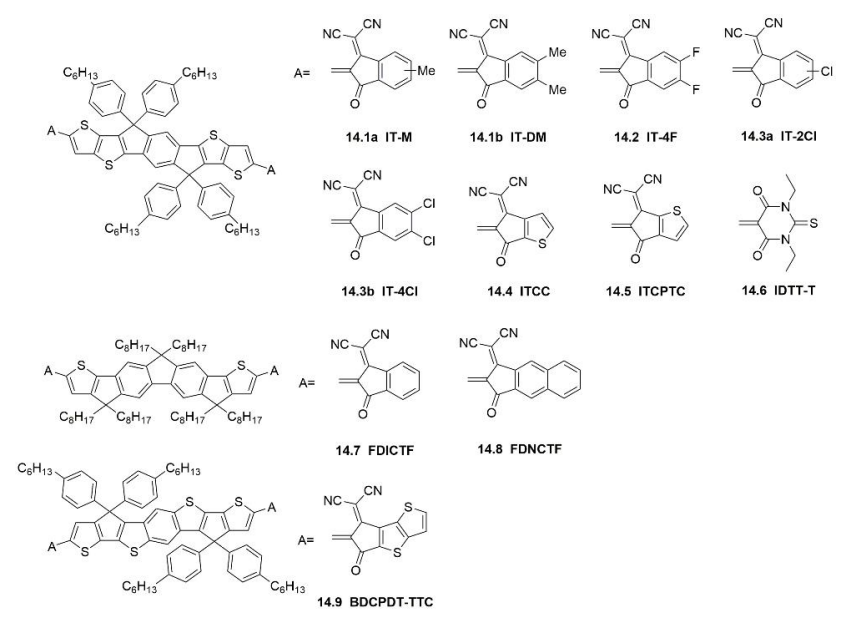

Figure 14 Chemical structures of NFAs with different endcapped units.

Zhao et al. ${ }^{[68]}$ designed and synthesized PBDB-T-SF and IT-4F (Figure 14, 14.2) through fluorination strategy. The absorption range of IT-4F is further extended to the NIR region. Compared to non-fluorinated analogs, the HOMO/LUMO levels of PBDBT-SF and IT-4F are downshifted to $-5.40 /-3.60 \mathrm{eV}$ and $-5.66 /-4.14 \mathrm{eV}$, respectively. The lower frontier orbitals energy levels of PBDB-T-SF and IT-4F provide good chemical stability and large polarization. Moreover, their more ordered intermolecular arrangements in film makes a slightly improvement in hole mobility of PBDB-T-SF and electron mobility of IT-4F. The PBDB-T-SF/IT-4F-based device showed a high efficiency of $13.1 \%$, and an efficiency of over $12 \%$ can be obtained with a thickness of $100-200 \mathrm{~nm}$. Zhang et al. ${ }^{[69]}$ prepared two chloro- analogs IT-2Cl and IT-4Cl (Figure 14, 14.3a, 14.3b). Compared with IT-4F, the HOMO and LUMO energy levels are down-shifted to -5.71 and $-3.62 \mathrm{eV}$ for IT-4CI. In the film, IT-4Cl showed a red-shifted absorption edge at 840 $\mathrm{nm}$. Combining with PBDB-T-2F, IT-4Cl acceptor can deliver an average PCE of $13.18 \%$ with a high $J_{S C}$ of $22.67 \mathrm{~mA} / \mathrm{cm}^{2}$, a $V_{O C}$ of $0.79 \mathrm{~V}$ and a FF of 0.752 .

Replacing the benzene unit of the INCN with a thiophene ring, ITCC (Figure 14, 14.4) was reported by Yao et al. ${ }^{[70]}$ ITCC possessed strong intermolecular $\pi-\pi$ interactions and excellent charge transporting capability in solid film. The electron donating effect of thiophene also raised the LUMO energy level of the molecule. TEM and AFM images confirmed the larger domains in the bulk of the PBDB-T/ITCC blend film, and the bimolecular recombination is more efficiently suppressed in the
ITCC-based device. The PBDB-T/ITCC device shows a maximum PCE of $11.4 \%$ with an impressive $V_{\text {OC }}$ of $1.01 \mathrm{~V}$, a $J_{S C}$ of $15.9 \mathrm{~mA} / \mathrm{cm}^{2}$ and a FF of 0.71 . Meanwhile, the PSC device based on PBDB-T/ITIC shows a lower PCE of $10.6 \%$ with a $V_{O C}$ of $0.93 \mathrm{~V}$, a $J_{\mathrm{SC}}$ of $17.0 \mathrm{~mA} / \mathrm{cm}^{2}$ and a $F F$ of 0.67 under the same processing conditions and the same PBDB-T batch.

ITCPTC (Figure 14, 14.5) is an isomer of ITCC designed by Xie et al. ${ }^{[71]}$ ITCPTC shows a slight smaller band gap, more planar structure than ITIC, with a small dihedral angle of $1.24^{\circ}$ between CPTCN and IDTT units. The enhanced phase separation gives rise to improved efficiencies, charge generation and charge transport, benefiting from the better electron delocalization of CPTCN moiety and stronger intermolecular interactions induced by sulfur-sulfur interaction. Using ITCPTC as the acceptor and wide-bandgap polymer PBT1-EH as the donor, a PCE of $11.8 \%$ is achieved with a remarkably enhanced FF of 0.751 , a near $20 \%$ boost in PCE with respect to the ITIC-based control device.

IDTT-T (Figure 14, 14.6) is a medium-bandgap non-fullerene acceptor developed by $\mathrm{He}$ et al. ${ }^{[72]}$ The $N, N^{\prime}$-diethylthiobarbituric acid (TBA) unit shows weakened electron withdrawing ability. As a result, the variation of $\mathrm{HOMO}$ levels was negligible between IDTT-T and ITIC. The LUMO energy level of IDTT-T is $0.25 \mathrm{eV}$ higher than ITIC. In the blend film with PTB7-Th, the electron mobility of ITIC is one order of magnitude lower than that of IDTT-T. The very similar patterns for the ITIC and IDTT-T blend films also suggested that the end groups in NFAs have little impact on molecular orientation and packing behavior. The OPV devices using PTB7-Th:IDTT-T as the active layer shows a high $V_{O C}$ over $1 \mathrm{~V}$, together with a high $J_{\text {sc }}$ up to $18.0 \mathrm{~mA} / \mathrm{cm}^{2}$, and a high PCE reaching $11.8 \%$.

In 2017, Feng et al. ${ }^{[73]}$ introduced (2-3-oxo-2,3-dihydro$1 \mathrm{H}$-cyclopenta[b]naphthalen-1-ylidene)-malononitrile (NINCN) to fluorine-dicyclopentathiophene core. Compared with its analog FDICTF (Figure 14, 14.7), FDNCTF (Figure 14, 14.8) exhibits stronger absorption in the region of $550-760 \mathrm{~nm}$ with a red-shifted and higher maximum extinction coefficient at 697 $\mathrm{nm}$. When a larger size of end groups is introduced, the intermolecular end-to-end $\pi-\pi$ interaction and molecular packing of FDNCTF makes a fundamental change. A quite strong (100) lamellar diffraction peak is seen at $0.32 \AA^{-1}$ with an interlamellar distance of $19.5 \AA$ in the in-plane direction; a sharp $\pi-\pi$ stacking (010) diffraction peak is seen at $1.82 \AA^{-1}$ in the out-of-plane direction with a d-spacing of $3.4 \AA$. Therefore, structural change from INIC to NINIC led to a new molecular ordering that turn an amorphous solid into a highly ordered thin film. Non-fullerene PSCs using FDNCTF and polymer PBDB-T demonstrated a high PCE of $11.2 \%$ with a $V_{O C}$ of $0.93 \mathrm{~V}$, a $J_{S C}$ of $16.5 \mathrm{~mA} / \mathrm{cm}^{2}$, and a $F F$ of $72.7 \%$, which is much higher than the PCE of $10.06 \%$ for the corresponding FDICTF-based device.

Recently, Chang et al. ${ }^{[74]}$ develop a novel end-capped unit TTC, which is obtained by replacing the benzene ring in IC with a more electron-rich thieno[3,2-b]thiophene unit. BDCPDTTTC (Figure 14, 14.9) exhibits a high lying LUMO energy level $(-3.78 \mathrm{eV})$ and additional optical transition at the short wavelengths around $300-500 \mathrm{~nm}$. Compared to the standard BDCPDT-IC device with a PCE of $9.33 \%$, the BDCPDT-TTCbased device gives an impressive efficiency of $10.29 \%$ with an enhanced $V_{\mathrm{OC}}$ of $0.94 \mathrm{~V}$ and $\mathrm{a} J_{\mathrm{SC}}$ of $17.72 \mathrm{~mA} / \mathrm{cm}^{2}$.

\section{A-T-D-m-A type NFA}

Conjugated $\pi$ units are often introduced to A-D-A structure, which usually give a more efficient intramolecular charge 


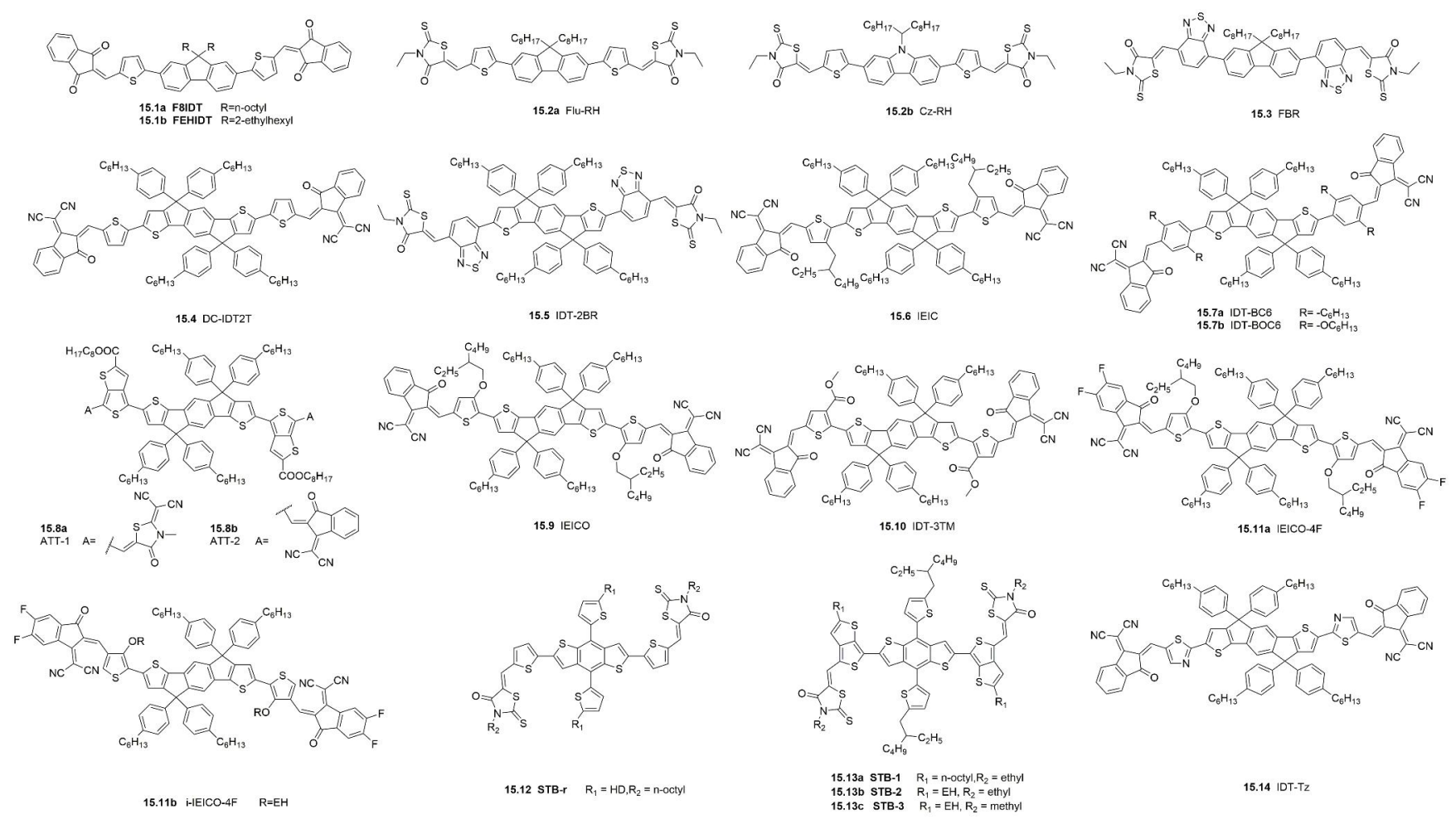

Figure 15 Chemical structures of A-m-D-ா-A type NFAs.

transfer (ICT) from the donor to the acceptor. The m-conjugated bridges are mostly dominated by thiophene derivatives owing to their excellent electrical properties and ease of functionalization. Moreover, stabilization of quinoidal form not only lowers the bandgap of related conjugated systems but also makes the overall backbone more coplanar.

Winzenberg et al. ${ }^{[75]}$ reported two NFAs named F8IDT (Figure 15, 15.1a) and FEHIDT (Figure15, 15.1b) in 2013. The acceptor unit $1 \mathrm{H}$-indene-1,3-dione is on the same plane with the $\pi$ bridge thiophene; the twist angle between the donor alkyl substituted fluorene and the plane is about 20 degrees. The onset of the UV/Vis absorbance are 2.00 and $2.10 \mathrm{eV}$, for FEHIDT and F8IDT, respectively. The optimized PCE for a device based on FEHIDT (2.43\%) and P3HT is around $30 \%$ higher than that obtained for F8IDT.

Flu-RH (Figure 15, 15.2a) and Cz-RH (Figure 15, 15.2b) based on carbazole and fluorene were synthesized with 3-ethylrhodanine by Kim et al. in 2014. ${ }^{[76]}$ The optical gaps of $\mathrm{Cz}-\mathrm{RH}$ and Flu-RH films are 2.05 and $2.10 \mathrm{eV}$, respectively. Flu-RH had a higher EQE intensity than that of $\mathrm{Cz}-\mathrm{RH}$ in the region of $350-800 \mathrm{~nm}$. The high-lying LUMO levels of Cz-RH and Flu-RH ensure a high open circuit voltage of the device. The best P3HT/Flu-RH device showed a PCE of $3.08 \%$ with a high $V_{O C}$ up to $1.03 \mathrm{~V}$.

Based on the rigid dialkyl substituted fluorene as donor core, 3-ethylrhodanine as electron accepting group, and 2,1,3-benzothiadiazole as $\pi$-spacer, Holliday et al. ${ }^{[77]}$ reported FBR acceptor (Figure 15, 15.3) with absorption range from 400 to $600 \mathrm{~nm}$. It is worth mentioning that the molar extinction of FBR is an order of magnitude higher than PCBM. A large dihedral angle of $35^{\circ}$ is calculated between the fluorene core and the benzothiadiazole unit. This makes an advantage to prevent the growth of large acceptor domains. Benefiting of above factors, the device based on FBR and P3HT gave a PCE of $4.1 \%$.

Bai et al. ${ }^{[78]}$ reported a novel acceptor DC-IDT2T (Figure 15, 15.4) based on IDT core with 1,1-dicyanomethylene-3indanone as electron-withdrawing end groups and thiophene
T-bridges. The donor and acceptor moieties formed a near flat plane, while the phenyl groups on the IDT exhibited a dihedral angle of ca. $115^{\circ}$ to the backbone plane. The HOMO and LUMO levels of the DC-IDT2T were estimated to be -5.43 and $-3.85 \mathrm{eV}$. The acceptor exhibited good thermal stability with decomposition temperature (5\% weight-loss) at $365{ }^{\circ} \mathrm{C}$ in nitrogen atmosphere. The PSC based on a blended film of PBDTTT-C-T/DC-IDT2T exhibited a broad photoresponse extending from 300 to $850 \mathrm{~nm}$, relatively high and balanced hole mobility $\left(2.0 \times 10^{-3} \mathrm{~cm}^{2} \cdot \mathrm{V}^{-1} \cdot \mathrm{s}^{-1}\right)$ and electron mobility $\left(1.5 \times 10^{-3} \mathrm{~cm}^{2} \cdot \mathrm{V}^{-1} \cdot \mathrm{s}^{-1}\right)$, and the best PSC achieved a $V_{O C}$ of $0.90 \mathrm{~V}$, a $J_{\mathrm{SC}}$ of $8.33 \mathrm{~mA} \cdot \mathrm{cm}^{-2}, F F$ of $52.3 \%$, and a PCE of $3.93 \%$ without any post-treatment.

Wu et al. ${ }^{[79]}$ developed a planar electron acceptor IDT-2BR (Figure 15, 15.5) using IDT unit as core, BR unit as endcapping groups and 2,1,3-benzothiadiazole as m-spacer. The optical bandgap of IDT-2BR estimated from the absorption edge of the thin film is $1.68 \mathrm{eV}$. The fullerene-free PSCs based on P3HT/IDT-2BR $(1: 0.6, w / w)$ blended films processed PCEs as high as $5.12 \%$ with $3 \% \mathrm{CN}$ additive.

In 2015, Lin et al. ${ }^{[80]}$ introduced 2-ethylhexyl at the 3 position of thiophene as spacer to contrast the acceptor IEIC (Figure 15, 15.6). The IEIC exhibited good thermal stability, strong absorption in the range of $500-750 \mathrm{~nm}$ with an extinction coefficient of $1.1 \times 10^{5} \mathrm{M}^{-1} \cdot \mathrm{cm}^{-1}$ (at $672 \mathrm{~nm}$ ). Its deep LUMO energy level of $-3.82 \mathrm{eV}$ is close to those of fullerene acceptors. Introducing the PDIN interlayer into the PSCs based on the blends of the IEIC and a low-bandgap polymer donor PTB7-Th showed a PCE up to $6.31 \%$.

It is worth mentioning that the research work done by Liu et al. in 2017. They adopt non-covalent conformational locking as design strategy to endow acceptor molecules with a good planarity in solid state together with a good solubility in solutions. Based on such a design strategy, bis(alkoxy)substituted or dialkyl-substituted benzene ring as conformational control units were added between the IDT and cyanoindanones. ${ }^{[81]}$ As a result, the dihedral angles between the 


\section{General Chemistry}

1,4-phenylene unit in the main chain and its neighboring units exhibit a big difference. The two dihedral angles for the simplified IDT-BC6 (Figure 15, 15.7a) molecule are $47^{\circ}$ and $36^{\circ}$; whereas the two dihedral angles are reduced to $9^{\circ}$ and $1^{\circ}$ for the simplified IDTBOC6 (Figure 15, 15.7b) molecule. IDTBC6 based device gave PCE of $2.28 \%$ with a $V_{\mathrm{OC}}$ of $0.92 \mathrm{~V}$, a $J_{\mathrm{SC}}$ of $5.63 \mathrm{~mA} / \mathrm{cm}^{2}$, and $F F$ of $44.2 \%$. In comparison, IDT-BOC6 based device exhibited PCE of $8.49 \%$ with a higher $V_{O C}$ of 1.01 $\mathrm{V}$, a $J_{\mathrm{SC}}$ of $15.35 \mathrm{~mA} / \mathrm{cm}^{2}$, and a $F F$ of $54.7 \%$. After the addition of $1 \%$ DIO, the PCE was further enhanced to $9.60 \%$ with a higher $J_{\mathrm{sc}}$ value of $17.52 \mathrm{~mA} / \mathrm{cm}^{2}$.

Two thieno[3,4-b]thiophene-based electron acceptors ATT-1 (Figure 15, 15.8a) and ATT-2 (Figure 15, 15.8b) have been designed and synthesized by Liu et al. ${ }^{[2,83]}$ Due to the difference of the acceptor unit, the orientation of the $\pi$ bridge is also different. The carbonyl group in the $\mathrm{DCl}$ and the sulfur atom in the $\pi$ bridge can form an interaction, which is enhanced by the planarity of the molecule. $\mathrm{DCl}$ has a stronger electronwithdrawing capability, the absorption edge of ATT-2 extends to $900 \mathrm{~nm}$ and obtained a lower optical energy gap $1.34 \mathrm{eV}$ than ATT-1. By combining with PTB7-Th, the as-cast PSCs yield PCEs of up to $9.58 \%$ with a $F F$ of 0.63 , a $V_{\text {OC }}$ of $0.73 \mathrm{~V}$, and a very high $J_{\mathrm{SC}}$ of $20.75 \mathrm{~mA} / \mathrm{cm}^{2}$.

Different from IEIC, the IEICO (Figure 15, 15.9) molecule was introduced an alkoxy group on thiophene. ${ }^{[84]}$ IEICO exhibited a much higher HOMO level than IEIC but a similar LUMO level. Thus, the solid film of IEICO had an absorption onset of $925 \mathrm{~nm}$ and an $E_{\mathrm{g}}^{\mathrm{opt}}$ of $1.34 \mathrm{eV}$, which is $0.16 \mathrm{eV}$ smaller than that of IEIC $(1.50 \mathrm{eV})$. After optimizing the device fabrication conditions, the PBDTTT-E-T/IEICO-based device showed a PCE of $8.4 \%$ with a $J_{S C}$ of $17.7 \mathrm{~mA} / \mathrm{cm}^{2}$, which is much higher than that of the PBDTTT-E-T/IEIC-based device (4.9\%).

IDT-3MT (Figure 15, 15.10) was synthesized through the thiophene-3-carboxylate bridging indacenodithiophene and 2-(3-oxo-2,3-dihydroinden-1-ylidene)-malononitrile. ${ }^{[85]}$ Attribu- ted to the strong intermolecular interaction of IDT-3MT, the acceptor showed relatively low-lying energy levels and a red-shifted absorption spectrum compared to IDT-T bearing thiophene bridges. The dihedral angle between the thiophene and the core unit was found to $15.09^{\circ}$ for the IDT-3MT. The PSCs based on as-cast PBDB-T/IDT-3MT blend film exhibited the highest PCE of $8.4 \%$ with a $V_{O C}$ of $0.95 \mathrm{~V}$ and a $J_{S C}$ of 14.43 $\mathrm{mA} / \mathrm{cm}^{2}$, benefiting from prominent face-on orientation and fine internal morphology of the blend film.

Wang et al. designed a pair of main-chain substituted isomer IEICO-4F (Figure 15, 15.11a) and i-IEICO-4F (Figure 15, 15.11b). They found that $i-I E I C O-4 F$ exhibits twisted main-chain configuration, resulting in blue-shift of absorption edge to 797 $\mathrm{nm}$ in solid film, which possessed complementary absorption with J52. ${ }^{[86]}$ The J52/IEICO-4F blend thin film gave a correlation length of $20.3 \mathrm{~nm}$, and the J52/i-IEICO-4F blend film gave a correlation length of $12.8 \mathrm{~nm}$. Meanwhile, a domain size of 25.8 and $18.5 \mathrm{~nm}$ for J52/IEICO-4F and J52/i-IEICO-4F blend films, respectively. A PCE of $13.18 \%$ with a $V_{O C}$ of $0.849 \mathrm{~V}$, a $J_{S C}$ of $22.86 \mathrm{~mA} / \mathrm{cm}^{2}$, and a FF of $67.9 \%$ were recorded in J52/i-IEICO4F-based PSCs.

$\mathrm{Xu}$ et al. ${ }^{[87]}$ prepared a series of 2-Alkylthieno[3,4-b]thiophene (T34bT) based acceptors. All the acceptor showed the same absorption maximum at $625 \mathrm{~nm}$, with high coefficients of $1.12 \times 10^{5} \mathrm{M}^{-1} \cdot \mathrm{cm}^{-1}$. In comparison with a thiophene-bridged molecule STB-r (Figure 15, 15.12), STB-n (Figure 15, 15.13) molecules had better absorption coefficients and broadened absorption spectra. The PCEs of STB-3 could be increased to $8.47 \%$ after SVA annealing with a $J_{\mathrm{SC}}$ of $13.03 \mathrm{~mA} / \mathrm{cm}^{2}$ and a FF of $71 \%$. Using PFN as cathode interlayer, the PCE can be improved to $9.26 \%$.

Yu et al. ${ }^{[88]}$ developed a novel IDT-based small molecular acceptor IDT-Tz (Figure 15, 15.14) using electron deficient thiazole as $\pi$-bridges to replace the thiophene of IDT-T. IDT-Tz exhibit highly planar con-formation, and the $\mathrm{N} \cdots \mathrm{S}$ distance of
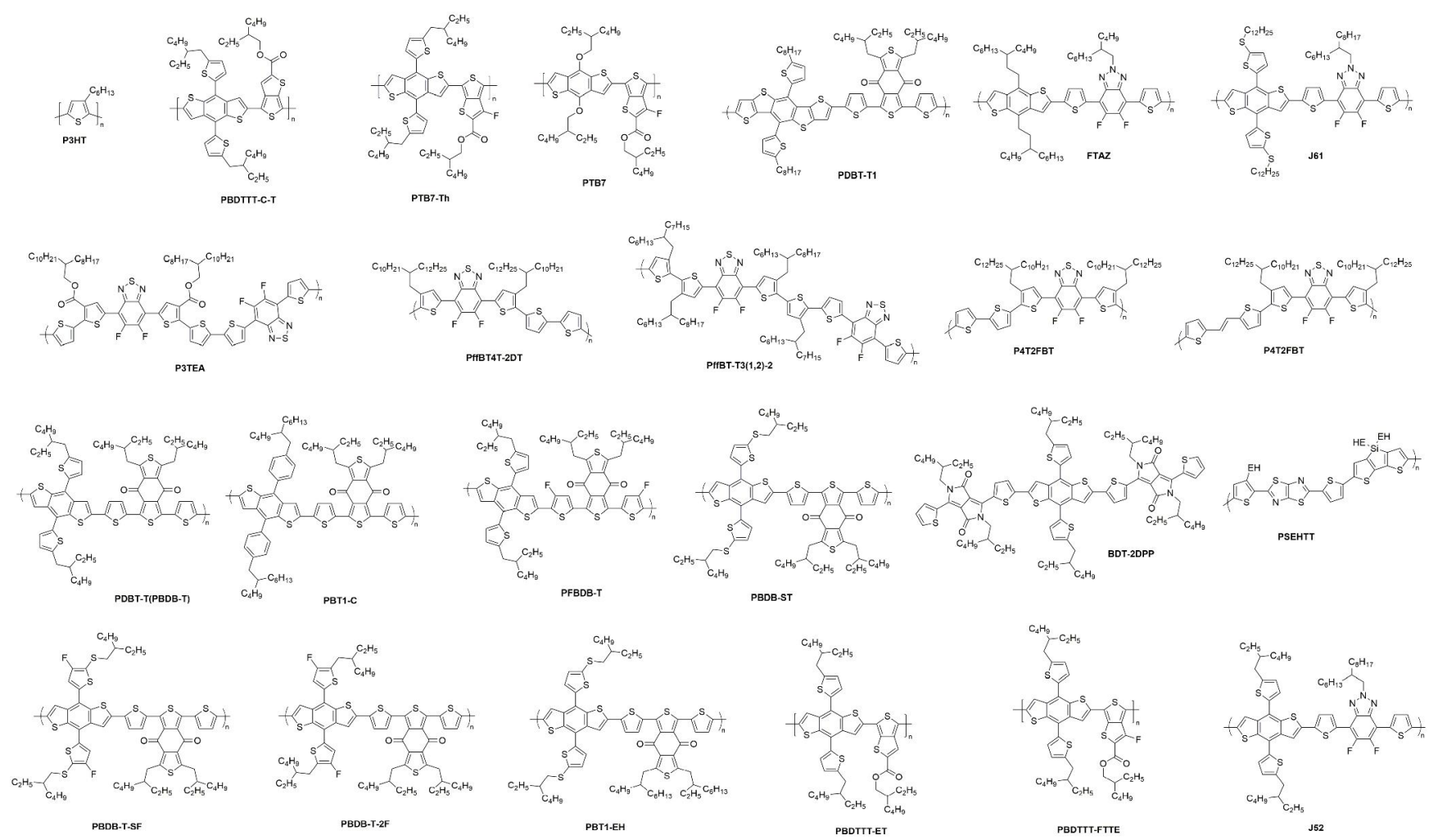

Figure 16 Chemical structures of the polymer donors mentioned in this review. 
$2.89 \AA$ is within the van der Waals radius $(\sim 3.5 \AA)$. Thus, the devices have a broad photoresponse range of $350-800 \mathrm{~nm}$. The EQE of IDT-Tz based device is over $70 \%$ across the range of $550-750 \mathrm{~nm}$, demonstrating an efficient photoelectron conversion process occurred. The introduction of the thiazole into IDT-Tz can reduce both HOMO $(-5.62 \mathrm{eV})$ and LUMO $(-4.09 \mathrm{eV})$ energy levels. Upon addition of $1.5 \% \mathrm{CN}$, a maximum PCE of $8.52 \%$ was achieved with a $V_{O C}$ of $0.88 \mathrm{~V}$, a $J_{\mathrm{SC}}$ of $13.67 \mathrm{~mA} / \mathrm{cm}^{2}$ and a $F F$ of $70.8 \%$ using PBDB-T as donor.

\section{Conclusions}

This review provides tentatively a comprehensive summary on the molecular engineering of NFAs for highly efficient PSCs, including PDI-based acceptors, A-D-A conjugated acceptors as well as A-ח-D-ா-A type acceptors. The examples presented in this review show clearly the structural diversity and high efficiency of non-fullerene acceptors, so that the tendency replacing fullerene-based acceptor with NFAs can be predictable in the field of organic photovoltaics. Herein, the successful instances will guide our molecule design for developing new NFA materials, meanwhile, these exciting results can also motivate the researches to explore novel building blocks and molecular design strategies. Certainly, there are some barriers we need to step over on the promising way; the issues include: (i) enhancing the structural stability and the morphology stability of NFAs in operating PSCs device is primary task, (ii) reducing the synthetic cost is expected, (iii) avoiding structure homogenization in NFAs molecular design are noticeable problems for researchers. With the further development of molecular engineering, it is believed that the NFA molecules would play increasingly important roles in next-generation solar cells.

\section{Acknowledgement}

This work was financially supported by the Science and Technology Project of Shenyang City (No. 18-013-0-26) and the Open Project of Key Laboratory for Organic Electronics and Information Displays (No. 201703).

\section{References}

[1] Tang, C. W. Appl. Phys. Lett. 1986, 48, 183.

[2] Yu, G.; Gao, J.; Hummelen, J. C.; Wudl, F.; Heeger, A. J. Science 1995, 270, 1789.

[3] Sariciftci, N. S.; Smilowitz, L.; Heeger, A. J.; Wudl, F. Science 1992, 258, 1474.

[4] Benten, H.; Mori, D.; Ohkita, H.; Ito, S. J. Mater. Chem. A 2016, 4, 5340

[5] Cai, Y.; Huo, L.; Sun, Y. Adv. Mater. 2017, 29, 1605437.

[6] He, Y.; Li, Y. PCCP 2011, 13, 1970.

[7] Nielsen, C. B.; Holliday, S.; Chen, H.-Y.; Cryer, S. J.; McCulloch, I. Acc. Chem. Res. 2015, 48, 2803.

[8] He, Y.; Chen, H.-Y.; Hou, J.; Li, Y. J. Am. Chem. Soc. 2010, 132, 1377.

[9] Zhang, S.; Ye, L.; Hou, J. Adv. Energy Mater. 2016, 6, 1502529.

[10] Zhou, Y.; Ding, L.; Shi, K.; Dai, Y.-Z.; Ai, N.; Wang, J.; Pei, J. Adv. Mater. 2012, 24, 957

[11] Yan, Q.; Zhou, Y.; Zheng, Y.-Q.; Pei, J.; Zhao, D. Chem. Sci. 2013, 4, 4389.

[12] Brunetti, F. G.; Gong, X.; Tong, M.; Heeger, A. J.; Wudl, F. Angew. Chem. Int. Ed. 2010, 49, 532.

[13] Lin, Y.; Wang, J.; Zhang, Z.-G.; Bai, H.; Li, Y.; Zhu, D.; Zhan, X. Adv. Mater. 2015, 27, 1170
[14] Xiao, Z.; Jia, X.; Ding, L. Chin. Sci. Bull. 2017, 62, 1562.

[15] Elumalai, N. K.; Uddin, A. Energ. Environ. Sci. 2016, 9, 391.

[16] Zhan, X.; Facchetti, A.; Barlow, S.; Marks, T. J.; Ratner, M. A.; Wasielewski, M. R.; Marder, S. R. Adv. Mater. 2010, 23, 268.

[17] Huang, C.; Barlow, S.; Marder, S. R. J. Org. Chem. 2011, 76, 2386.

[18] Matthews, R.; Swisher, J.; Hutchins, K. M.; Pentzer, E. B. Chem. Mater. 2018, 30, 3571

[19] Ozdal, D.; Aydinlik, N. P.; Bodapati, J. B.; Icil, H. Photoch. Photobio. Sci. 2017, 16, 262

[20] Sung, J.; Kim, P.; Fimmel, B.; Würthner, F.; Kim, D. Nat. Commun. 2015, 6, 8646

[21] Rajaram, S.; Shivanna, R.; Kandappa, S. K.; Narayan, K. S. J. Phys. Chem. Lett. 2012, 3, 2405.

[22] Jiang, W.; Ye, L.; Li, X.; Xiao, C.; Tan, F.; Zhao, W.; Hou, J.; Wang, Z. Chem. Commun. 2014, 50, 1024.

[23] Sun, D.; Meng, D.; Cai, Y.; Fan, B.; Li, Y.; Jiang, W.; Huo, L.; Sun, Y.; Wang, Z. J. Am. Chem. Soc. 2015, 137, 11156.

[24] Meng, D.; Sun, D.; Zhong, C.; Liu, T.; Fan, B.; Huo, L.; Li, Y.; Jiang, W.; Choi, H.; Kim, T.; Kim, J. Y.; Sun, Y.; Wang, Z.; Heeger, A. J. J. Am. Chem. Soc. 2016, 138, 375.

[25] Wu, C.-H.; Chueh, C.-C.; Xi, Y.-Y.; Zhong, H.-L.; Gao, G.-P.; Wang, Z.-H.; Pozzo, L. D.; Wen, T.-C.; Jen, A. K. Y. Adv. Funct. Mater. 2015, 25, 5326.

[26] Wang, H.; Chen, L.; Xiao, Y. J. Mater. Chem. A 2017, 5, 22288.

[27] Zhang, X.; Lu, Z.; Ye, L.; Zhan, C.; Hou, J.; Zhang, S.; Jiang, B.; Zhao, Y.; Huang, J.; Zhang, S.; Liu, Y.; Shi, Q.; Liu, Y.; Yao, J. Adv. Mater. 2013, 25, 5791.

[28] Lin, Y.; Wang, J.; Dai, S.; Li, Y.; Zhu, D.; Zhan, X. Adv. Energy Mater. 2014, 4, 1400420

[29] Zhao, J.; Li, Y.; Zhang, J.; Zhang, L.; Lai, J. Y. L.; Jiang, K.; Mu, C.; Li, Z.; Chan, C. L. C.; Hunt, A.; Mukherjee, S.; Ade, H.; Huang, X.; Yan, H. J. Mater. Chem. A 2015, 3, 20108.

[30] Wang, J.; Yao, Y.; Dai, S.; Zhang, X.; Wang, W.; He, Q.; Han, L.; Lin, Y.; Zhan, X. J. Mater. Chem. A 2015, 3, 13000.

[31] Hadmojo, W. T.; Nam, S. Y.; Shin, T. J.; Yoon, S. C.; Jang, S.-Y.; Jung, I. H. J. Mater. Chem. A 2016, 4, 12308.

[32] Li, Z.; Jiang, K.; Yang, G.; Lai, J. Y. L.; Ma, T.; Zhao, J.; Ma, W.; Yan, H. Nat. Commun. 2016, 7, 13094.

[33] Lin, Y.; Wang, Y.; Wang, J.; Hou, J.; Li, Y.; Zhu, D.; Zhan, X. Adv. Mater. 2014, 26, 5137.

[34] Li, S.; Liu, W.; Li, C.-Z.; Liu, F.; Zhang, Y.; Shi, M.; Chen, H.; Russell, T. P. J. Mater. Chem. A 2016, 4, 10659.

[35] Liu, Y.; Mu, C.; Jiang, K.; Zhao, J.; Li, Y.; Zhang, L.; Li, Z.; Lai, J. Y. L.; Hu, H.; Ma, T.; Hu, R.; Yu, D.; Huang, X.; Tang, B. Z.; Yan, H. Adv. Mater. 2014, 27, 1015.

[36] Liu, Y.; Lai, J. Y. L.; Chen, S.; Li, Y.; Jiang, K.; Zhao, J.; Li, Z.; Hu, H.; Ma, T.; Lin, H.; Liu, J.; Zhang, J.; Huang, F.; Yu, D.; Yan, H. J. Mater. Chem. A 2015, 3, 13632.

[37] Liu, S.-Y.; Wu, C.-H.; Li, C.-Z.; Liu, S.-Q.; Wei, K.-H.; Chen, H.-Z.; Jen, A. K. Y. Adv. Sci. 2015, 2, 1500014

[38] Lee, J.; Singh, R.; Sin, D. H.; Kim, H. G.; Song, K. C.; Cho, K. Adv. Mater. 2015, 28, 69.

[39] Zhong, Y.; Trinh, M. T.; Chen, R.; Wang, W.; Khlyabich, P. P.; Kumar, B.; Xu, Q.; Nam, C.-Y.; Sfeir, M. Y.; Black, C.; Steigerwald M. L.; Loo, Y.-L.; Xiao, S.; Ng, F.; Zhu, X. Y.; Nuckolls, C. J. Am. Chem. Soc. 2014, 136, 15215.

[40] Zhong, H.; Wu, C.-H.; Li, C.-Z.; Carpenter, J.; Chueh, C.-C.; Chen, J.-Y.; Ade, H.; Jen, A. K. Y. Adv. Mater. 2015, 28, 951.

[41] Hartnett, P. E.; Matte, H. S. S. R.; Eastham, N. D.; Jackson, N. E.; Wu, Y.; Chen, L. X.; Ratner, M. A.; Chang, R. P. H.; Hersam, M. C. Wasielewski, M. R.; Marks, T. J. Chem. Sci. 2016, 7, 3543.

[42] Li, S.; Liu, W.; Li, C.-Z.; Lau, T.-K.; Lu, X.; Shi, M.; Chen, H. J. Mater. Chem. A 2016, 4, 14983.

[43] Zhang, J.; Li, Y.; Huang, J.; Hu, H.; Zhang, G.; Ma, T.; Chow, P. C Y.; Ade, H.; Pan, D.; Yan, H. J. Am. Chem. Soc. 2017, 139, 16092.

[44] Wang, B.; Liu, W.; Li, H.; Mai, J.; Liu, S.; Lu, X.; Li, H.; Shi, M.; Li, 


\section{General Chemistry}

C.-Z.; Chen, H. J. Mater. Chem. A 2017, 5, 9396.

[45] Gao, G.; Liang, N.; Geng, H.; Jiang, W.; Fu, H.; Feng, J.; Hou, J.; Feng, X.; Wang, Z. J. Am. Chem. Soc. 2017, 139, 15914.

[46] Lin, Y.; He, Q.; Zhao, F.; Huo, L.; Mai, J.; Lu, X.; Su, C.-J.; Li, T.; Wang, J.; Zhu, J.; Sun, Y.; Wang, C.; Zhan, X. J. Am. Chem. Soc. 2016, 138, 2973.

[47] Xiao, Z.; Liu, F.; Geng, X.; Zhang, J.; Wang, S.; Xie, Y.; Li, Z.; Yang, H.; Yuan, Y.; Ding, L. Chin. Sci. Bull. 2017, 62, 1331.

[48] Ma, Y.; Zhang, M.; Yan, Y.; Xin, J.; Wang, T.; Ma, W.; Tang, C.; Zheng, Q. Chem. Mater. 2017, 29, 7942.

[49] Wang, W.; Yan, C.; Lau, T.-K.; Wang, J.; Liu, K.; Fan, Y.; Lu, X.; Zhan, X. Adv. Mater. 2017, 29, 1701308.

[50] Zhang, J.; Yan, C.; Wang, W.; Xiao, Y.; Lu, X.; Barlow, S.; Parker, T. C.; Zhan, X.; Marder, S. R. Chem. Mater. 2018, 30, 309

[51] Li, C.; Xie, Y.; Fan, B.; Han, G.; Yi, Y.; Sun, Y. J. Mater. Chem. C 2018, 6, 4873

[52] Zhao, W.; Qian, D.; Zhang, S.; Li, S.; Inganäs, O.; Gao, F.; Hou, J. Adv. Mater. 2016, 28, 4734

[53] Kan, B.; Feng, H.; Wan, X.; Liu, F.; Ke, X.; Wang, Y.; Wang, Y.; Zhang, H.; Li, C.; Hou, J.; Chen, Y. J. Am. Chem. Soc. 2017, 139, 4929.

[54] Qiu, N.; Zhang, H.; Wan, X.; Li, C.; Ke, X.; Feng, H.; Kan, B.; Zhang, H.; Zhang, Q.; Lu, Y.; Chen, Y. Adv. Mater. 2016, 29, 1604964.

[55] Wang, P.; Fan, H.; Zhang, C.; Zhu, X. Mater. Chem. Front. 2018, 2, 136.

[56] Zhu, J.; Ke, Z.; Zhang, Q.; Wang, J.; Dai, S.; Wu, Y.; Xu, Y.; Lin, Y.; Ma, W.; You, W.; Zhan, X. Adv. Mater. 2017, 30, 1704713.

[57] Li, T.; Dai, S.; Ke, Z.; Yang, L.; Wang, J.; Yan, C.; Ma, W.; Zhan, X. Adv. Mater. 2018, 30, 1705969

[58] Xiao, Z.; Jia, X.; Li, D.; Wang, S.; Geng, X.; Liu, F.; Chen, J.; Yang, S.; Russell, T. P.; Ding, L. Chin. Sci. Bull. 2017, 62, 1494.

[59] Dai, S.; Zhao, F.; Zhang, Q.; Lau, T.-K.; Li, T.; Liu, K.; Ling, Q.; Wang, C.; Lu, X.; You, W.; Zhan, X. J. Am. Chem. Soc. 2017, 139, 1336.

[60] Li, Y.; Liu, X.; Wu, F.-P.; Zhou, Y.; Jiang, Z.-Q.; Song, B.; Xia, Y.; Zhang, Z.-G.; Gao, F.; Inganäs, O.; Li, Y.; Liao, L.-S. J. Mater. Chem. A 2016, 4, 5890 .

[61] Li, Y.; Qian, D.; Zhong, L.; Lin, J.-D.; Jiang, Z.-Q.; Zhang, Z.-G.; Zhang, Z.; Li, Y.; Liao, L.-S.; Zhang, F. Nano Energy 2016, 27, 430.

[62] Jia, B.; Dai, S.; Ke, Z.; Yan, C.; Ma, W.; Zhan, X. Chem. Mater. 2018, 30, 239

[63] Lin, Y.; Zhao, F.; He, Q.; Huo, L.; Wu, Y.; Parker, T. C.; Ma, W.; Sun, Y.; Wang, C.; Zhu, D.; Heeger, A. J.; Marder, S. R.; Zhan, X. J. Am. Chem. Soc. 2016, 138, 4955.

[64] Yang, Y.; Zhang, Z.-G.; Bin, H.; Chen, S.; Gao, L.; Xue, L.; Yang, C.; Li, Y. J. Am. Chem. Soc. 2016, 138, 15011.

[65] Zhang, C. e.; Feng, S.; Liu, Y.; Hou, R.; Zhang, Z.; Xu, X.; Wu, Y.; Bo, Z. ACS Appl. Mater. Interfaces 2017, 9, 33906.

[66] Fei, Z.; Eisner, F. D.; Jiao, X.; Azzouzi, M.; Röhr, J. A.; Han, Y.; Shahid, M.; Chesman, A. S. R.; Easton, C. D.; McNeill, C. R.; Anthopoulos, T. D.; Nelson, J.; Heeney, M. Adv. Mater. 2018, 30, 1705209.
[67] Li, S.; Ye, L.; Zhao, W.; Zhang, S.; Mukherjee, S.; Ade, H.; Hou, J. Adv. Mater. 2016, 28, 9423.

[68] Zhao, W.; Li, S.; Yao, H.; Zhang, S.; Zhang, Y.; Yang, B.; Hou, J. J. Am. Chem. Soc. 2017, 139, 7148.

[69] Zhang, H.; Yao, H.; Hou, J.; Zhu, J.; Zhang, J.; Li, W.; Yu, R.; Gao B.; Zhang, S.; Hou, J. Adv. Mater. 2018, 30, 1800613.

[70] Yao, H.; Ye, L.; Hou, J.; Jang, B.; Han, G.; Cui, Y.; Su, G. M.; Wang C.; Gao, B.; Yu, R.; Zhang, H.; Yi, Y.; Woo, H. Y.; Ade, H.; Hou, J. Adv. Mater. 2017, 29, 1700254.

[71] Xie, D.; Liu, T.; Gao, W.; Zhong, C.; Huo, L.; Luo, Z.; Wu, K.; Xiong, W.; Liu, F.; Sun, Y.; Yang, C. Solar RRL 2017, 1, 1700044

[72] He, B.; Yang, B.; Kolaczkowski, M. A.; Anderson, C. A.; Klivansky, L. M.; Chen, T. L.; Brady, M. A.; Liu, Y. ACS Energy Lett. 2018, 3 1028.

[73] Feng, H.; Qiu, N.; Wang, X.; Wang, Y.; Kan, B.; Wan, X.; Zhang, M.; Xia, A.; Li, C.; Liu, F.; Zhang, H.; Chen, Y. Chem. Mater. 2017, 29, 7908.

[74] Chang, S.-L.; Cao, F.-Y.; Huang, W.-C.; Huang, P.-K.; Huang, K.-H.; Hsu, C.-S.; Cheng, Y.-J. ACS Energy Lett. 2018, 3, 1722.

[75] Winzenberg, K. N.; Kemppinen, P.; Scholes, F. H.; Collis, G. E.; Shu, Y.; Birendra Singh, T.; Bilic, A.; Forsyth, C. M.; Watkins, S. E. Chem. Commun. 2013, 49, 6307.

[76] Kim, Y.; Song, C. E.; Moon, S.-J.; Lim, E. Chem. Commun. 2014 $50,8235$.

[77] Holliday, S.; Ashraf, R. S.; Nielsen, C. B.; Kirkus, M.; Röhr, J. A.; Tan, C.-H.; Collado-Fregoso, E.; Knall, A.-C.; Durrant, J. R.; Nelson, J.; McCulloch, I. J. Am. Chem. Soc. 2015, 137, 898.

[78] Bai, H.; Wang, Y.; Cheng, P.; Wang, J.; Wu, Y.; Hou, J.; Zhan, X. J. Mater. Chem. A 2015, 3, 1910

[79] Wu, Y.; Bai, H.; Wang, Z.; Cheng, P.; Zhu, S.; Wang, Y.; Ma, W.; Zhan, X. Energ. Environ. Sci. 2015, 8, 3215

[80] Lin, Y.; Zhang, Z.-G.; Bai, H.; Wang, J.; Yao, Y.; Li, Y.; Zhu, D.; Zhan, X. Energ. Environ. Sci. 2015, 8, 610.

[81] Liu, Y.; Zhang, Z.; Feng, S.; Li, M.; Wu, L.; Hou, R.; Xu, X.; Chen X.; Bo, Z. J. Am. Chem. Soc. 2017, 139, 3356

[82] Liu, F.; Zhou, Z.; Zhang, C.; Vergote, T.; Fan, H.; Liu, F.; Zhu, X. J. Am. Chem. Soc. 2016, 138, 15523.

[83] Liu, F.; Zhou, Z.; Zhang, C.; Zhang, J.; Hu, Q.; Vergote, T.; Liu, F.; Russell, T. P.; Zhu, X. Adv. Mater. 2017, 29, 1606574.

[84] Yao, H.; Chen, Y.; Qin, Y.; Yu, R.; Cui, Y.; Yang, B.; Li, S.; Zhang, K.; Hou, J. Adv. Mater. 2016, 28, 8283.

[85] Park, S. H.; Park, G. E.; Choi, S.; Kim, Y. U.; Park, S. Y.; Park, C. G.; Cho, M. J.; Choi, D. H. J. Mater. Chem. C 2018, 6, 7549.

[86] Wang, W.; Zhao, B.; Cong, Z.; Xie, Y.; Wu, H.; Liang, Q.; Liu, S.; Liu, F.; Gao, C.; Wu, H.; Cao, Y. ACS Energy Lett. 2018, 3, 1499.

[87] Xu, S.; Zhou, Z.; Fan, H.; Ren, L.; Liu, F.; Zhu, X.; Russell, T. P. J. Mater. Chem. A 2016, 4, 17354.

[88] Yu, S.; Chen, Y.; Yang, L.; Ye, P.; Wu, J.; Yu, J.; Zhang, S.; Gao, Y.; Huang, H. J. Mater. Chem. A 2017, 5, 21674

Received September 28, 2018 Accepted October 29, 2018 